\title{
3D Born-Infeld gravity and supersymmetry
}

\section{Eric Bergshoeff and Mehmet Ozkan}

Institute for Particle Physics and Gravity, University of Groningen, Nijenborgh 4, 9747 AG Groningen, The Netherlands

E-mail: e.a.bergshoeff@rug.nl, m.ozkan@rug.nl

ABSTRACT: We construct the most general parity-even higher-derivative $\mathcal{N}=1$ off-shell supergravity model in three dimensions with a maximum of six derivatives. Excluding terms quadratic in the curvature tensor with two explicit derivatives and requiring the absence of ghosts in a linearized approximation around an $\mathrm{AdS}_{3}$ background, we find that there is a unique supersymmetric invariant which we call supersymmetric 'cubic extended' New Massive Gravity. The purely gravitational part of this invariant is in agreement with an earlier analysis based upon the holographic c-theorem and coincides with an expansion of Born-Infeld gravity to the required order.

Our results lead us to propose an expression for the bosonic part of off-shell $\mathcal{N}=1$ Born-Infeld supergravity in three dimensions that is free of ghosts. We show that different truncations of a perturbative expansion of this expression gives rise to the bosonic part of (i) Einstein supergravity; (ii) supersymmetric New Massive Gravity and (iii) supersymmetric 'cubic extended' New Massive Gravity.

KEYwords: Classical Theories of Gravity, Supergravity Models

ARXIV EPRINT: 1405.6212 


\section{Contents}

1 Introduction 1

2 Poincaré supergravity with a cosmological constant 4

2.1 Weyl and matter multiplets 4

$\begin{array}{lll}2.2 & \text { Poincaré supergravity with a cosmological constant } & 6\end{array}$

3 Supersymmetric four-derivative invariants $\quad 9$

$\begin{array}{ll}3.1 \text { The supersymmetric } R^{2} \text { invariant } & 10\end{array}$

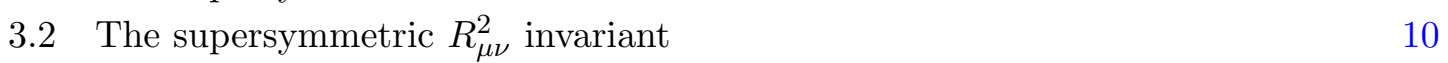

$\begin{array}{lll}3.3 & \text { Supersymetric } R S^{n} \text { invariants } & 12\end{array}$

$\begin{array}{lll}3.4 & \text { Supersymmetric new massive gravity } & 13\end{array}$

4 Supersymmetric six-derivative invariants $\quad 14$

$\begin{array}{lll}4.1 & \text { Diagonal invariants } & 14\end{array}$

$\begin{array}{lll}4.2 & \text { Off-diagonal invariants } & 18\end{array}$

5 Towards 3D Born-Infeld supergravity $\quad 21$

5.1 Supersymmetric cubic extended NMG 21

$\begin{array}{lll}5.2 & \text { Towards 3D Born-Infeld supergravity } 22\end{array}$

6 Conclusions $\quad 24$

\section{Introduction}

Higher-derivative supergravity Lagrangians have received considerable attention in recent years. There are several reasons for this. First of all, such models have been used to calculate corrections to the BPS black hole entropy in a given setting [1]. More generally, such higher-derivative models provide an interesting test of the range of validity of the AdS/CFT correspondence [2]. Higher-derivative gravity models have also occurred in discussions of the holographic c-theorem [3]. More recently, a specific four-derivative gravity model in three dimensions, called New Massive Gravity (NMG) [4], has been introduced as the parity-even version of Topologically Massive Gravity (TMG) [5].

An example of a 3D higher-derivative gravity theory with an infinite number of higherderivative terms is the so-called Born-Infeld (BI) gravity theory [6] which is a particular example of the Born-Infeld-Einstein theories studied in [7]. It was observed in [6] that upon making a truncation, that keeps terms with at most four derivatives in a perturbative expansion of this BI gravity theory, one ends up with the ghost-free NMG model [4]. Furthermore, by making a different truncation that retains terms with at most six derivatives one ends up with what we will call the 'cubic extended' NMG model $[6,8,9]$. 
The supersymmetric extension of NMG was given in [10,11]. The construction of such an invariant turns out to be more subtle than anticipated. It is not difficult to construct the $\mathcal{N}=1$ supersymmetric extension of the Einstein-Hilbert term with a cosmological constant and of the Ricci tensor and Ricci scalar squared terms ${ }^{1}$ that make up the NMG invariant. When setting the cosmological constant to zero and linearizing the model around the maximally supersymmetric Minkowski ${ }_{3}$ background, one finds that the model is ghostfree as expected. However, a complication arises when taking the cosmological constant into account. The Ricci scalar squared and the Ricci tensor squared invariants include couplings between the gravitational field and the auxiliary scalar $S$ that is part of the $\mathcal{N}=1$ supergravity multiplet in such a way that ghosts are re-introduced in the linearized approximation around $\mathrm{AdS}_{3}$.

To be specific, assigning the gravitational field a mass dimension zero and the auxiliary scalar $S$ a mass dimension one, the bosonic part of the most general $\mathcal{N}=1$ supersymmetric invariant of the form $1 / m^{2} \times$ [terms of mass dimension four] is given by $[10,11]$

$$
\begin{aligned}
e^{-1} \mathcal{L}_{\mathrm{SQuad}}=\frac{1}{m^{2}}[ & a_{1} R_{\mu \nu} R^{\mu \nu}+a_{2} R^{2}-\left(6 a_{1}+16 a_{2}\right) \partial_{\mu} S \partial^{\mu} S \\
& \left.+\left(4 a_{1}+12 a_{2}+\frac{3}{10} a_{3}\right) R S^{2}+\left(12 a_{1}+36 a_{2}+a_{3}\right) S^{4}\right],
\end{aligned}
$$

where $m$ is a mass parameter and $a_{1}, a_{2}, a_{3}$ are dimensionless parameters representing three different supersymmetric invariants. To obtain the complete model, the above invariant has to be added to the supersymmetric completion of the Einstein-Hilbert term with a cosmological constant. We will call the $R S^{2}$ term that contains both the curvature tensor and the auxiliary scalar 'off-diagonal'.

When four-derivative terms are added to the Poincaré supergravity, the auxiliary scalar $S$ becomes dynamical, and the dynamical scalar forms a multiplet with the gravitational scalar ghost, and the gamma trace of the gravitino field strength $\gamma \cdot \mathcal{R}$ where $\mathcal{R}^{\mu}=\epsilon^{\mu \nu \rho} \nabla_{\nu} \psi_{\rho}$. Therefore, the kinetic term for the auxiliary scalar $S$ in (1.1) is unacceptable since it would introduce an extra ghost degree of freedom. The coefficient in front of this kinetic term vanishes precisely for the NMG combination $a_{2}=-\frac{3}{8} a_{1}$, in which case the $\left(\frac{1}{2}, 0,0\right)$ ghost multiplet becomes infinitely heavy and decouples from the spectrum. That still leaves us with the off-diagonal $R S^{2}$ term. It is easily seen that, given the complete model, upon linearization around an $\mathrm{AdS}_{3}$ background with a constant value of the auxiliary scalar ${ }^{2}$ the presence of the $R S^{2}$ term leads, after elimination of the auxiliary scalar perturbation, to extra terms quadratic in the curvature tensor thereby upsetting the ghost-free NMG combination. Therefore, to cancel the unwanted $R S^{2}$ term, we need to take $a_{3}=\frac{5}{3} a_{1}$. Taking $a_{1}=1$, we thus end up with the ghost-free combination

$$
e^{-1} \mathcal{L}_{\text {ghost-free }}=\frac{1}{m^{2}}\left[\left(R_{\mu \nu} R^{\mu \nu}-\frac{3}{8} R^{2}\right)+\frac{1}{6} S^{4}\right],
$$

that is part of the supersymmetric NMG model $[10,11]$.

\footnotetext{
${ }^{1}$ Remember that in three dimensions the Riemann tensor is is proportional to the Ricci tensor.

${ }^{2}$ We use here that such configurations are solutions of the complete model.
} 
In this paper we wish to push the above analysis one level further and consider the supersymmetric completion of the most general terms of the form $1 / m^{4} \times$ [terms of mass dimension six]. We do not consider terms of the form $R \square R$ or $R_{\mu \nu} \square R^{\mu \nu}$ since such terms lead to models with two massive gravitons one of which is a ghost [12]. It turns out that one can write down 15 terms of mass dimension 6 that involve the metric tensor and/or the auxiliary scalar. Considering only purely gravitational terms that are cubic in the curvature tensor, we find that a supersymmetric completion involves only 11 of these terms ${ }^{3}$ Three of them are purely gravitational, six are off-diagonal and two are purely auxiliary:

$$
\begin{array}{ll}
R_{\mu \nu} R^{\nu \rho} R_{\rho}^{\mu}, R^{3}, R R_{\mu \nu} R^{\mu \nu}: & \text { purely gravitational (3), } \\
R^{2} S^{2}, R S^{4}, S^{2} R_{\mu \nu} R^{\mu \nu}, & \\
R_{\mu \nu} \partial^{\mu} S \partial^{\nu} S, R \partial_{\mu} S \partial^{\mu} S, R S \square S: & \text { off-diagonal (6), } \\
S^{2} \partial_{\mu} S \partial^{\mu} S, S^{6}: & \text { purely auxiliary (2). }
\end{array}
$$

We find that the supersymmetric completion of these terms contains 8 parameters corresponding to the 8 supersymmetric invariants that we will construct in this work. One of the terms in the above expression $\left(S^{2} \partial_{\mu} S \partial^{\mu} S\right)$ is un-acceptable since in a linearization it gives rise to a dynamical $S$ in the spectrum, which, once again forms a ghost multiplet with the gravitational scalar ghost and the gamma trace of the gravitino field strength. Furthermore, all of the 6 off-diagonal terms are un-acceptable since each of them would upset, after linearization around an $\mathrm{AdS}_{3}$ background, the ghost-free NMG combination. This imposes a total of 7 restrictions on our 8 parameters thereby leading to a unique solution which is given in eq. (5.3). This equation contains all terms of mass dimension 6 of what we will call the supersymmetric 'cubic extended' NMG model.

We find that the purely gravitational terms of the supersymmetric 'cubic extended' NMG model precisely coincides with a specific truncation of BI gravity that keeps terms with at most 6 derivatives. Given our results for the supersymmetric completion of this specific truncation of the BI gravity model we are led to propose an expression for the bosonic part of the $\mathcal{N}=1 \mathrm{BI}$ supergravity theory, see eq. (5.6). Upon making different truncations this $\mathcal{N}=1 \mathrm{BI}$ supergravity model reproduces the bosonic sector of (i) Einstein supergravity; (ii) supersymmetric New Massive Gravity and (iii) supersymmetric 'cubic extended' New Massive Gravity. We will comment about some further features of this proposed BI supergravity model.

The organisation of this paper is as follows. As a warming up exercise we will briefly review in section 2 how to construct Poincaré supergravity with a cosmological constant using the superconformal tensor calculus. In section 3 we will consider the supersymmetric completion of the most general terms of mass dimension 4 thereby re-producing the supersymmetric NMG model $[10,11]$. The purpose of this section is to prepare the reader for section 4 where we will push the analysis one level further and consider the supersymmetric extension of the most general terms of mass dimension 6 . The results obtained in

\footnotetext{
${ }^{3}$ We do not consider the terms $(\square S)^{2}$ and $S \epsilon_{\mu \nu \rho} R^{\mu}{ }_{\sigma} \nabla^{\rho} R^{\nu \sigma}$ since these terms only occur as part of a supersymmetric $R \square R$ or $R_{\mu \nu} \square R^{\mu \nu}$ invariant which we exclude.
} 
this section will enable us to construct in section 5 the supersymmetric completion of cubic extended NMG. In a separate subsection, based on our experience with the supersymmetric completion of the NMG and the cubic extended NMG models, we propose an expression for the bosonic part of $\mathcal{N}=1$ Born-Infeld supergravity. We will comment about our results in the Conclusions.

\section{Poincaré supergravity with a cosmological constant}

The purpose of this section is to familiarize the reader with the conformal tensor calculus and construct Poincaré supergravity with a cosmological constant. In the first subsection, we introduce the $3 D, \mathcal{N}=1$ Weyl multiplet and the basic matter multiplets we will need in this paper, i.e. the scalar and Yang-Mills multiplet. In the second subsection, using these basic ingredients, we construct the Poincaré supergravity theory with a cosmological constant. For an early reference on $3 D$ supergravity, see [13]. Below, we will make extensive use of the superconformal $D=3, \mathcal{N}=1$ superconformal tensor calculus constructed in $[14-16]$.

\subsection{Weyl and matter multiplets}

Our starting point is the $3 D, \mathcal{N}=1$ Weyl multiplet, with $2+2$ off-shell degrees of freedom, whose independent gauge fields are the Dreibein $e_{\mu}{ }^{a}$, the gravitino $\psi_{\mu}$ and the dilatation gauge field $b_{\mu}{ }^{4}$ These gauge fields transform under dilatations $D$, special conformal transformations $K, Q$-supersymmetry and $S$-supersymmetry, with parameters $\Lambda_{D}, \Lambda_{K}, \epsilon$ and $\eta$, respectively, as follows [15]:

$$
\begin{aligned}
\delta e_{\mu}{ }^{a} & =\frac{1}{2} \bar{\epsilon} \gamma^{a} \psi_{\mu}-\Lambda_{D} e_{\mu}{ }^{a} \\
\delta \psi_{\mu} & =\mathcal{D}_{\mu}(\omega) \epsilon+\gamma_{\mu} \eta-\frac{1}{2} \Lambda_{D} \psi_{\mu} \\
\delta b_{\mu} & =-\frac{1}{2} \bar{\epsilon} \phi_{\mu}+\frac{1}{2} \bar{\eta} \psi_{\mu}+\partial_{\mu} \Lambda_{D}+2 \Lambda_{K \mu}
\end{aligned}
$$

where

$$
\mathcal{D}_{\mu}(\omega) \epsilon=\left(\partial_{\mu}+\frac{1}{2} b_{\mu}+\frac{1}{4} \omega_{\mu}^{a b} \gamma_{a b}\right) \epsilon
$$

is covariant with respect to dilatations and Lorentz transformations. The expressions for the spin-connection field $\omega_{\mu}^{a b}$, the special conformal gauge field $f_{\mu}{ }^{a}$ and the Ssupersymmetry gauge field $\phi_{\mu}$ in terms of the independent gauge fields $e_{\mu}{ }^{a}, \psi_{\mu}$ and $b_{\mu}$ are given by

$$
\begin{aligned}
\omega_{\mu}^{a b} & =2 e^{\nu[a} \partial_{[\mu} e_{\nu]}^{b]}-e^{\nu[a} e^{b] \sigma} e_{\mu c} \partial_{\nu} e_{\sigma}^{c}+2 e_{\mu}{ }^{[a} b^{b]}+\frac{1}{2} \bar{\psi}_{\mu} \gamma^{[a} \psi^{b]}+\frac{1}{4} \bar{\psi}^{a} \gamma_{\mu} \psi^{b}, \\
\phi_{\mu} & =-\gamma^{a} \widehat{R}_{\mu a}^{\prime}(Q)+\frac{1}{4} \gamma_{\mu} \gamma^{a b} \widehat{R}_{a b}^{\prime}(Q), \\
f_{\mu}{ }^{a} & =-\frac{1}{2} \widehat{R}_{\mu}^{\prime a}(M)+\frac{1}{8} e_{\mu}{ }^{a} \widehat{R}^{\prime}(M),
\end{aligned}
$$

where $\widehat{R}_{\mu}{ }^{a}(M)=\widehat{R}_{\mu \nu}{ }^{a b}(M) e^{\nu}{ }_{b}$ and $\widehat{R}(M)=e_{a}{ }^{\mu} \widehat{R}_{\mu}{ }^{a}(M)$. Here $\widehat{R}_{\mu \nu}(Q)$ and $\widehat{R}_{\mu \nu}{ }^{a b}(M)$ are the super-covariant curvatures of $Q$-supersymmetry and Lorentz transformations $M$,

\footnotetext{
${ }^{4} a=0,1$ is a Lorentz index and $\mu=0,1$ is a curved index. We use two-component Majorana spinors.
} 


\begin{tabular}{|c|c|c|c|c|}
\hline multiplet & field & type & off-shell & $D$ \\
\hline Weyl & $e_{\mu}{ }^{a}$ & dreibein & 4 & 1 \\
& $\psi_{\mu}$ & gravitino & 4 & $\frac{1}{2}$ \\
\hline Scalar & $\mathcal{A}$ & scalar & 1 & $\omega_{\mathcal{A}}$ \\
& $\chi$ & spinor & 2 & $\omega_{\mathcal{A}}+\frac{1}{2}$ \\
& $\mathcal{F}$ & auxiliary & 1 & $\omega_{\mathcal{A}}+1$ \\
\hline \multirow{2}{*}{ Yang-Mills } & $A_{\mu}^{I}$ & gauge field & 2 & 0 \\
& $\varphi^{I}$ & gaugino & 2 & $\frac{3}{2}$ \\
\hline
\end{tabular}

Table 1. This table summarizes some properties of the basic multiplets of the $3 D$ superconformal tensor calculus. The fourth column indicates the number of off-shell degrees of freedom represented by the field. The last column specifies the dilatation weight. For the Yang-Mills multiplet we have only counted the off-shell degrees of freedom for one value of the Lie algebra index $I$.

respectively. The expressions for these curvatures are given by

$$
\begin{aligned}
\widehat{R}_{\mu \nu}(Q) & =2 \partial_{[\mu} \psi_{\nu]}+\frac{1}{2} \omega_{[\mu}^{a b} \gamma_{a b} \psi_{\nu]}+b_{[\mu} \psi_{\nu]}-2 \gamma_{[\mu} \phi_{\nu]} \\
\widehat{R}_{\mu \nu}{ }^{a b}(M) & =2 \partial_{[\mu} \omega_{\nu]}^{a b}+2 \omega_{[\mu}^{a c} \omega_{\nu] c}^{b}+8 f_{[\mu}^{[a} e_{\nu]}^{b]}-\bar{\phi}_{[\mu} \gamma^{a b} \psi_{\nu]} \cdot
\end{aligned}
$$

The prime in $\widehat{R}^{\prime}(M)$ indicates that we have omitted the $f_{\mu}{ }^{a}$ term in the expression for the full curvature $\widehat{R}(M)$. Similarly, the prime in $\widehat{R}^{\prime}(Q)$ indicates that we have omitted the $\phi_{\mu}$ term in the expression for the full curvature $\widehat{R}(Q)$. In table 1 , see below, we have collected a few basic properties of the Weyl multiplet.

We next introduce the basic superconformal matter multiplets. First, we consider the scalar multiplet with $2+2$ off-shell degrees of freedom. This multiplet consists of a physical scalar $\mathcal{A}$, a Majorana fermion $\chi$ and an auxiliary scalar $\mathcal{F}$. We assign the scalar $\mathcal{A}$ an arbitrary dilatation weight $\omega_{\mathcal{A}}$. The weights of the other fields, together with some other properties of the scalar multiplet, can be found in table 1. The $Q$ and $S$ transformations of the scalar multiplet component fields are given by [15]

$$
\begin{aligned}
\delta \mathcal{A} & =\frac{1}{4} \bar{\epsilon} \chi, \\
\delta \chi & =\not{D} \mathcal{A} \epsilon-\frac{1}{4} F \epsilon-2 \mathcal{A} \omega_{\mathcal{A}} \eta, \\
\delta \mathcal{F} & =-\bar{\epsilon} \not{D} \chi-2\left(\omega_{\mathcal{A}}-\frac{1}{2}\right) \bar{\eta} \chi,
\end{aligned}
$$

where the supercovariant derivatives of $\mathcal{A}$ and $\chi$ are given by

$$
\begin{aligned}
& \mathcal{D}_{\mu} \mathcal{A}=\left(\partial_{\mu}-\omega_{\mathcal{A}} b_{\mu}\right) \mathcal{A}-\frac{1}{4} \bar{\psi}_{\mu} \chi \\
& \mathcal{D}_{\mu} \chi=\left(\partial_{\mu}-\left(\omega_{\mathcal{A}}+\frac{1}{2}\right) b_{\mu}+\frac{1}{4} \omega_{\mu}{ }^{a b} \gamma_{a b}\right) \chi-\not D \mathcal{A} \psi_{\mu}+\frac{1}{4} F \psi_{\mu}+2 \mathcal{A} \omega_{\mathcal{A}} \phi_{\mu}
\end{aligned}
$$

In the rest of this paper we will need four special scalar multiplets with specific choices of the weight $\omega_{\mathcal{A}}$. It is convenient to give names to the components of these four multiplets, see table 2 . 


\begin{tabular}{|c|c|c|}
\hline name & $\omega_{\mathcal{A}}$ & components \\
\hline Auxiliary Scalar Multiplet & 2 & $(Z, \Omega, F)$ \\
Curvature Scalar Multiplet & $\frac{3}{2}$ & $(\xi, \varphi, M)$ \\
Compensating Scalar Multiplet & $\frac{1}{2}$ & $(\phi, \lambda, S)$ \\
Neutral Scalar Multiplet & 0 & $(\sigma, \psi, N)$ \\
\hline
\end{tabular}

Table 2. This table indicates the nomenclature for the four scalar multiplets that will play a special role in the superconformal tensor calculus we use in this paper.

We finally consider the superconformal Yang-Mills multiplet. This multiplet consists of a vector field $A_{\mu}^{I}$, with zero Weyl weight, and a Majorana spinor $\varphi^{I}$, where $I$ is a Lie algebra index, see table 1 . This multiplet has $2+2$ off-shell degrees of freedom per value of the Lie algebra index. The supersymmetry transformations of the component fields are given by

$$
\begin{aligned}
\delta A_{\mu}^{I} & =-\bar{\epsilon} \gamma_{\mu} \varphi^{I} \\
\delta \varphi^{I} & =\frac{1}{8} \gamma^{\mu \nu} \widehat{F}_{\mu \nu}^{I} \epsilon,
\end{aligned}
$$

where the supercovariant field strength is defined as

$$
\widehat{F}_{\mu \nu}^{I}=2 \partial_{[\mu} A_{\nu]}^{I}+g f_{J L}^{I} A_{\mu}^{J} A_{\nu}^{L}+2 \bar{\psi}_{[\mu} \gamma_{\nu]} \varphi^{I}
$$

\subsection{Poincaré supergravity with a cosmological constant}

In this subsection we construct a Poincaré supergravity theory, together with a cosmological constant, using the superconformal ingredients given in the previous subsection. Our starting point is the action for a general scalar multiplet coupled to conformal supergravity. To cancel the weight -3 of the $e=\operatorname{det} e_{\mu}{ }^{a}$ factor in the leading term, we need to consider an auxiliary scalar multiplet since its highest component $F$ has weight 3 . The Lagrangian corresponding to such an action is given by $[15]^{5}$

$$
e^{-1} \mathcal{L}_{\mathrm{Aux}}=F-\bar{\psi}_{\mu} \gamma^{\mu} \Omega-Z \bar{\psi}_{\mu} \gamma^{\mu \nu} \psi_{\nu}
$$

Before proceeding to the construction of the Poincaré supergravity action, we first discuss the multiplication rule for scalar multiplets and the construction of a kinetic multiplet for any given scalar multiplet. The multiplication rule states that two scalar multiplets can be multiplied together to form a third scalar multiplet. In component formalism, if we consider two scalar multiplets $\left(\mathcal{A}_{i}, \chi_{i}, \mathcal{F}_{i}\right), i=1,2$, with the conformal weight of the

\footnotetext{
${ }^{5}$ This is the full Lagrangian including the fermionic terms. From now on, to avoid the cluttering of complicated expressions, we adopt the convention that we only give the bosonic part of a supersymmetric Lagrangian unless we explicitly state that we include the fermions as we will do in a few cases. If needed, all fermionic terms can easily be restored by the superconformal tensor calculus.
} 
lowest component given by $\omega_{\mathcal{A}_{i}}$ a third scalar multiplet with components $\left(\mathcal{A}_{3}, \chi_{3}, \mathcal{F}_{3}\right)$ and weight $\omega_{\mathcal{A}_{3}}=\omega_{\mathcal{A}_{1}}+\omega_{\mathcal{A}_{2}}$ can be obtained by the following multiplication rules:

$$
\begin{aligned}
\mathcal{A}_{3} & =\mathcal{A}_{1} \mathcal{A}_{2}, \\
\chi_{3} & =\mathcal{A}_{1} \chi_{2}+\mathcal{A}_{2} \chi_{1}, \\
\mathcal{F}_{3} & =\mathcal{A}_{1} \mathcal{F}_{2}+\mathcal{A}_{2} \mathcal{F}_{1}+\bar{\chi}_{1} \chi_{2} .
\end{aligned}
$$

These rules can be inverted as follows:

$$
\begin{aligned}
\mathcal{A}_{1} & =\mathcal{A}_{3} \mathcal{A}_{2}^{-1} \\
\chi_{1} & =\mathcal{A}_{2}^{-1} \chi_{3}-\mathcal{A}_{3} \mathcal{A}_{2}^{-2} \chi_{2} \\
\mathcal{F}_{1} & =\mathcal{A}_{2}^{-1} \mathcal{F}_{3}-\mathcal{A}_{3} \mathcal{A}_{2}^{-2} \mathcal{F}_{2}-\mathcal{A}_{2}^{-2} \bar{\chi}_{2} \chi_{3}+\mathcal{A}_{3} \mathcal{A}_{2}^{-3} \bar{\chi}_{2} \chi_{2}
\end{aligned}
$$

Next, we discuss the idea of a kinetic multiplet of a given scalar multiplet. A kinetic multiplet is formed from the composite expressions of a scalar multiplet in which the dynamical scalar $\mathcal{A}$ and fermion $\chi$ have a kinetic term. In terms of components, given a scalar multiplet, indicated with the subscript $s$, a kinetic multiplet, denoted by the subscript $k$, is obtained by the following rules:

$$
\begin{aligned}
\mathcal{A}_{k}= & \omega_{\mathcal{A}_{s}} \mathcal{F}_{s}-\frac{1}{2}\left(\omega_{\mathcal{A}_{s}}-\frac{1}{2}\right) \mathcal{A}_{s}^{-1} \bar{\chi}_{s} \chi_{s} \\
\chi_{k}= & -4 \omega_{\mathcal{A}_{s}} \not \mathcal{D} \chi_{s}+4\left(\omega_{\mathcal{A}_{s}}-\frac{1}{2}\right) \mathcal{A}_{s}^{-1} \not \mathcal{D}_{s} \chi_{s}+\left(\omega_{\mathcal{A}_{s}}-\frac{1}{2}\right) \mathcal{A}_{s}^{-1} \mathcal{F}_{s} \chi_{s} \\
& +\frac{1}{2}\left(\omega_{\mathcal{A}_{s}}-\frac{1}{2}\right) \mathcal{A}_{s}^{-2} \chi_{s} \bar{\chi}_{s} \chi_{s} \\
\mathcal{F}_{k}= & 16 \omega_{\mathcal{A}_{s}} \square^{c} \mathcal{A}_{s}-16\left(\omega_{\mathcal{A}_{s}}-\frac{1}{2}\right) \mathcal{A}_{s}^{-1} \mathcal{D}_{\mu} \mathcal{A}_{s} \mathcal{D}^{\mu} \mathcal{A}_{s}+\left(\omega_{\mathcal{A}_{s}}-\frac{1}{2}\right) \mathcal{A}_{s}^{-1} \mathcal{F}_{s}^{2} \\
& -4\left(\omega_{\mathcal{A}_{s}}-\frac{1}{2}\right) \mathcal{A}_{s}^{-1} \bar{\chi}_{s} \not \mathcal{D}_{\chi_{s}}-\frac{1}{2}\left(\omega_{\mathcal{A}_{s}}-\frac{1}{2}\right) \mathcal{A}_{s}^{-2} \mathcal{F}_{s} \bar{\chi}_{s} \chi_{s} \\
& -\frac{1}{2}\left(\omega_{\mathcal{A}_{s}}-\frac{1}{2}\right) \mathcal{A}_{s}^{-3} \bar{\chi}_{s} \chi_{s} \bar{\chi}_{s} \chi_{s} .
\end{aligned}
$$

The superconformal d'Alambertian occurring in the expression for $\mathcal{F}_{k}$ is given by

$$
\square^{c} \mathcal{A}_{s}=\left(\partial^{a}-\left(\omega_{s}+1\right) b^{a}+\omega_{b}^{b a}\right) \mathcal{D}_{a} \mathcal{A}_{s}+2 \omega_{s} f_{a}^{a} \mathcal{A}_{s}-\frac{1}{4} \bar{\psi}_{a} \mathcal{D}^{a} \chi_{s}-\frac{1}{4} \bar{\phi}_{a} \gamma^{a} \chi_{s}
$$

For future reference, we notice that applying the kinetic multiplet formula (2.12) we obtain the following relation between a compensating multiplet $(\phi, \lambda, S)$ and a curvature multiplet $(\xi, \varphi, M)$ :

$$
\xi=S, \quad \varphi=-4 \not D \lambda, \quad M=16 \square^{c} \phi,
$$

where we have rescaled the composite formulae (2.12) with a factor of 2 . Notice that the difference between the conformal weights of the lowest components of the scalar and corresponding kinetic multiplets is $\omega_{k}-\omega_{s}=1$. Using the kinetic multiplet and the (inverse) multiplication rules, it is not difficult to obtain the Lagrangian for a scalar multiplet with conformal weight $\omega$ coupled to a compensating multiplet with components $(\phi, \lambda, S)$, see 
table 2. One first multiplies the scalar multiplet with its kinetic multiplet and, next, with appropriate powers of the compensating multiplet. One thus obtains the following result:

$$
\begin{aligned}
e^{-1} \mathcal{L}_{\omega}= & 16 \omega \phi^{2-4 \omega} \mathcal{A}_{\omega} \square^{c} \mathcal{A}_{\omega}-16\left(\omega-\frac{1}{2}\right) \phi^{2-4 \omega} \mathcal{D}_{\mu} \mathcal{A}_{\omega} \mathcal{D}^{\mu} \mathcal{A}_{\omega} \\
& +\phi^{2-4 \omega}\left(2 \omega-\frac{1}{2}\right) \mathcal{F}_{\omega}^{2}-\omega(4 \omega-2) \phi^{1-4 \omega} S \mathcal{A}_{\omega} \mathcal{F}_{\omega}
\end{aligned}
$$

As stated in footnote 5 from now on we only give the bosonic terms of a supersymmetric Lagrangian unless otherwise stated. The fermionic terms can be read off from the composite formulae (2.12).

With these general multiplication and kinetic multiplet construction rules in hand, it is relative straightforward to construct matter coupled $\mathcal{N}=1$ Poincaré supergravity models. Setting $\omega=\frac{1}{2}$ in the action formulae (2.15) and identifying the general scalar multiplet with the compensating scalar multiplet, we obtain the following Lagrangian describing the coupling of a compensating scalar multiplet to conformal supergravity

$$
e^{-1} \mathcal{L}_{\mathrm{SC}, \mathrm{R}}=16 \phi \square^{c} \phi+S^{2} .
$$

To obtain a Poincaré supergravity theory we gauge fix the superconformal transformations by imposing the following gauge fixing conditions

$$
\phi=-\frac{1}{2}, \quad \lambda=0, \quad b_{\mu}=0
$$

where the first choice fixes dilatation, the second fixes the S-supersymmetry and the last one fixes the special conformal transformations. These gauge fixing conditions result into the following decomposition rules

$$
\Lambda_{K \mu}=\frac{1}{4} \bar{\epsilon} \phi_{\mu}-\frac{1}{4} \bar{\eta} \psi_{\mu}, \quad \eta=\frac{1}{2} S \epsilon .
$$

We thus end up with a Poincaré multiplet consisting of a Dreibein $e_{\mu}{ }^{a}$, a gravitino $\psi_{\mu}$ and an auxiliary scalar $S$ :

$$
\text { Poincaré multiplet: } \quad\left(e_{\mu}{ }^{a}, \psi_{\mu}, S\right) \text {. }
$$

Substituting the gauge fixing conditions (2.17) into the Lagrangian (2.16), and rescaling with a factor of -2, we obtain the following Lagrangian for this Poincaré multiplet (including the fermionic terms):

$$
e^{-1} \mathcal{L}_{\mathrm{R}}=R-2 S^{2}-\bar{\psi}_{\mu} \gamma^{\mu \nu \rho} \nabla_{\nu}(\omega) \psi_{\rho}
$$

This Lagrangian is invariant under the following transformation rules;

$$
\begin{aligned}
\delta e_{\mu}{ }^{a} & =\frac{1}{2} \bar{\epsilon} \gamma^{a} \psi_{\mu}, \\
\delta \psi_{\mu} & =\nabla_{\mu}(\omega) \epsilon+\frac{1}{2} S \gamma_{\mu} \epsilon, \\
\delta S & =\frac{1}{4} \bar{\epsilon} \gamma^{\mu \nu} \psi_{\mu \nu}(\omega)-\frac{1}{4} S \bar{\epsilon} \gamma^{\mu} \psi_{\mu},
\end{aligned}
$$

where

$$
\nabla_{\mu}(\omega) \epsilon=\left(\partial_{\mu}+\frac{1}{4} \omega_{\mu}^{a b} \gamma_{a b}\right) \epsilon, \quad \psi_{\mu \nu}=\nabla_{[\mu}(\omega) \psi_{\nu]}
$$


This matches with the expressions given in [17]. We note that if we define a torsionful spin-connection as follows

$$
\Omega_{\mu}^{ \pm a b}=\omega_{\mu}^{a b}+S \epsilon_{\mu}^{a b}
$$

the supersymmetry transformations (2.22) can be rewritten as [10]

$$
\begin{aligned}
\delta e_{\mu}{ }^{a} & =\frac{1}{2} \bar{\epsilon} \gamma^{a} \psi_{\mu} \\
\delta \psi_{\mu} & =\nabla_{\mu}\left(\Omega^{-}\right) \epsilon \\
\delta S & =\frac{1}{4} \bar{\epsilon} \gamma^{\mu \nu} \psi_{\mu \nu}\left(\Omega^{-}\right)
\end{aligned}
$$

Finally, a supersymmetric cosmological constant can be added to the Poincaré Lagrangian (2.20) by multiplying four copies of the compensating multiplet:

$$
Z=\phi^{4}, \quad \Omega=4 \phi^{3} \lambda, \quad F=4 \phi^{3} S+6 \phi^{2} \bar{\lambda} \lambda .
$$

Using these expressions into the action formula (2.9), and gauge fixing via (2.17) and scaling the resulting Lagrangian with factor of -2 , we obtain the following supersymmetric cosmological constant Lagrangian (including the fermionic terms)

$$
e^{-1} \mathcal{L}_{\mathrm{C}}=S+\frac{1}{8} \bar{\psi}_{\mu} \gamma^{\mu \nu} \psi_{\nu}
$$

which can be added to the Poincaré Lagrangian (2.20). This finishes our review of the construction of Poincaré supergravity with a cosmological constant via superconformal methods.

\section{Supersymmetric four-derivative invariants}

In this section we review the construction of supersymmetric $R^{2}$ and $R_{\mu \nu}^{2}$ invariants by using superconformal techniques and applying various maps between the scalar, YangMills and Poincaré multiplets. We consider the following most general expression of mass dimension 4:

$$
e^{-1} \mathcal{L}^{(4)}=a_{1} R_{\mu \nu} R^{\mu \nu}+a_{2} R^{2}+a_{3} R S^{2}+a_{4} S \square S+a_{5} S^{4}
$$

As explained in the introduction, naively one would expect that a proper combination of a Ricci scalar squared and Ricci tensor squared supersymmetric invariant gives rise to the supersymmetric NMG model which should be ghost free. However, a spectrum analysis of $R^{2}$ and $R_{\mu \nu}^{2}$ extended Poincaré supergravity around $A d S_{3}$ reveals that the mere use of offshell $R^{2}$ and $R_{\mu \nu}^{2}$ invariants is not sufficient to obtain a ghost-free model [11]. In particular, to obtain a ghost-free model, one needs a third supersymmetric invariant to cancel the offdiagonal $R S^{2}$ term. We will show that, besides the $R^{2}$ and $R_{\mu \nu}^{2}$ invariants, indeed such a third supersymmetric off-diagonal invariant exists. Below we will consider the construction of these three different invariants one after the other. In the last subsection we will apply these results to construct the supersymmetric NMG model [10, 11]. 


\subsection{The supersymmetric $R^{2}$ invariant}

In this section, we construct the supersymmetric completion of the $R^{2}$ term. In order to achieve this, we first need to find the superconformal map from a neutral scalar multiplet to the Weyl multiplet. There may be various maps from different scalar multiplets to the Weyl multiplet. However, since the lowest component of the neutral multiplet carries no Weyl weight, it has the special feature that it can be multiplied to another scalar multiplet without changing its properties, but only gives rise to composite expressions, like in (2.10). This property plays a crucial role in the construction of higher derivative supergravity invariants later on. Considering a neutral scalar multiplet $(\sigma, \psi, N)$ and two compensating multiplets, $\left(\phi_{1}, \lambda_{1}, S_{1}\right)$ and $\left(\phi_{2}, \lambda_{2}, S_{2}\right)$, sequential use of the inverse multiplication rule (2.11) implies the following composite expressions

$$
\begin{aligned}
\sigma & =\phi_{1}^{-3} S_{2}, \\
\psi & =-4 \phi_{1}^{-3} \not \mathcal{D} \lambda_{2}-3 \phi_{1}^{-4} S_{2} \lambda_{1}, \\
N & =16 \phi_{1}^{-3} \square^{C} \phi_{2}-3 \phi_{1}^{-4} S_{1} S_{2}+12 \phi_{1}^{-4} \bar{\lambda}_{1} \not \mathcal{D} \lambda_{2}+6 \phi_{1}^{-5} S_{2} \bar{\lambda}_{1} \lambda_{2} .
\end{aligned}
$$

We note that this map reduces to

$$
(\sigma, \quad \psi, \quad N) \longleftrightarrow\left(S, \quad \gamma^{\mu \nu} \psi_{\mu \nu}\left(\Omega^{-}\right), \quad \widehat{R}\left(\Omega^{ \pm}\right)\right) .
$$

upon rescaling the map (3.2) with an overall $-1 / 8$ factor, setting the two compensating multiplets equal to each other and gauge fixing via (2.17). The gauge fixed result matches with the result of [10]. Here the torsionful Ricci scalar reads

$$
\widehat{R}\left(\Omega^{ \pm}\right)=R(\omega)+6 S^{2}+2 \bar{\psi}_{\mu} \gamma_{\nu} \psi^{\mu \nu}\left(\Omega^{-}\right)+\frac{1}{2} S \psi_{\mu} \gamma^{\mu \nu} \psi_{\nu}
$$

The conformal action for the neutral scalar multiplet can easily be obtained using the action formula (2.15). Setting $\omega=0$ in (2.15), gauge fixing according to (2.17), and multiplying the resulting action with an overall $-1 / 8$ factor, we obtain the following action for a Poincaré neutral scalar multiplet:

$$
e^{-1} \mathcal{L}_{\text {Neutral }}=-\partial_{\mu} \sigma \partial^{\mu} \sigma+\frac{1}{16} N^{2} .
$$

Using the map (3.3), we obtain the supersymmetric completion of the Ricci scalar squared action $[10]$

$$
e^{-1} \mathcal{L}_{R^{2}}=\frac{1}{16} R^{2}-\partial_{\mu} S \partial^{\mu} S+\frac{3}{4} R S^{2}+\frac{9}{4} S^{4}
$$

\subsection{The supersymmetric $R_{\mu \nu}^{2}$ invariant}

To construct a supersymmetric $R_{\mu \nu}^{2}$ invariant we consider a superconformal Yang-Mills multiplet, see table 1. After gauge-fixing the superconformal symmetries via eq. (2.17), one finds the following map between the Yang-Mills and supergravity Poincaré multiplets [10]:

$$
\left(\Omega_{\mu}^{+a b}, \quad \psi^{a b}\left(\Omega^{-}\right)\right) \longleftrightarrow \quad\left(A_{\mu}^{I}, \quad \varphi^{I}\right)
$$


We see that the map (3.7) associates the Yang-Mills field strength $F_{a b}^{I}$ to the torsionful Riemann tensor $R_{a b}{ }^{c d}\left(\Omega^{+}\right)$given by

$$
R_{\mu \nu}^{a b}\left(\Omega^{ \pm}\right)=R_{\mu \nu}^{a b}(\omega) \pm \frac{1}{2} S \bar{\psi}_{\mu} \gamma^{a b} \psi_{\nu} \pm 2 \partial_{[\mu} S \epsilon_{\nu]}^{a b}+2 S^{2} e_{[\mu}^{a} e_{\nu]}^{b} .
$$

Therefore, all we need to do in order to obtain a supersymmetric $R_{\mu \nu}^{2}$ invariant is to first construct a superconformal Yang-Mills action. After substituting the gauge-fixing conditions (2.17) into this action, one can simply obtain the supersymmetric completion of a $R_{\mu \nu}^{2}$ action, modulo a supersymmetric $R^{2}$ action, by applying the map (3.7), since in three dimensions the Riemann tensor reads

$$
R_{\mu \nu a b}=\epsilon_{\mu \nu \rho} \epsilon_{a b c} G^{\rho c}
$$

where $G_{\mu a}$ is the Einstein tensor.

For the construction of a superconformal Yang-Mills action, we turn to the action (2.9) for the auxiliary scalar multiplet. Since we are after the supergravity coupled Yang-Mills action, we wish to express the components of the auxiliary multiplet in terms of the components of the Yang-Mills multiplet thereby using the compensating multiplet to balance the conformal weights. We thus find the following composite expressions:

$$
\begin{aligned}
Z= & \phi^{-2} \bar{\varphi}^{I} \varphi^{I}, \\
\Omega= & -2 \phi^{-3} \lambda \bar{\varphi}^{I} \varphi^{I}-\phi^{-2} \gamma \cdot \widehat{F}^{I} \varphi^{I}, \\
F= & -\phi^{-2} \widehat{F}_{\mu \nu}^{I} \widehat{F}^{\mu \nu I}-2 \phi^{-3} S \bar{\varphi}^{I} \varphi^{I}-8 \phi^{-2} \bar{\varphi}^{I} \not \mathcal{D} \varphi^{I} \\
& -2 \phi^{-4} \bar{\lambda} \gamma \cdot \widehat{F} \varphi^{I}-3 \phi^{-4} \bar{\lambda} \lambda \bar{\varphi}^{I} \varphi^{I} .
\end{aligned}
$$

Upon substituting these composite expressions into the action (2.9), using the gauge fixing conditions (2.17), and multiplying the resulting action with an overall $-1 / 16$ factor, we find the following supersymmetric Yang-Mills action (including the fermionic terms)

$$
\begin{aligned}
e^{-1} \mathcal{L}_{Y M}= & -\frac{1}{4} F_{\mu \nu}^{I} F^{\mu \nu I}-2 \bar{\varphi}^{I} \not \nabla \varphi^{I}+\frac{1}{2} \bar{\psi}_{\rho} \gamma^{\mu \nu} \gamma^{\rho} \varphi^{I} F_{\mu \nu}^{I}+S \bar{\varphi}^{I} \varphi^{I} \\
& -\frac{1}{2} \bar{\varphi}^{I} \varphi^{I} \bar{\psi}_{\mu} \psi^{\mu}+\frac{1}{8} \bar{\varphi}^{I} \varphi^{I} \bar{\psi}_{\mu} \gamma^{\mu \nu} \psi_{\nu}
\end{aligned}
$$

Applying the map (3.7) between the Yang-Mills and Poincaré multiplets we obtain the following supersymmetric completion of a $R_{\mu \nu}^{2}+R^{2}$ action [10]:

$$
e^{-1} \mathcal{L}_{R_{\mu \nu}^{2}+R^{2}}=-R_{\mu \nu} R^{\mu \nu}+\frac{1}{4} R^{2}+2 \partial_{\mu} S \partial^{\mu} S-R S^{2}-3 S^{4} .
$$

Finally, subtracting the $R^{2}$ part, see eq. (3.6), we obtain the following $R_{\mu \nu}^{2}$ action

$$
e^{-1} \mathcal{L}_{R_{\mu \nu}^{2}}=R_{\mu \nu} R^{\mu \nu}-6 \partial_{\mu} S \partial^{\mu} S+4 R S^{2}+12 S^{4} .
$$

Before proceeding, we wish to discuss an alternative way of constructing the Ricci tensor squared invariant in order to prepare the reader for the construction method of the $R_{\mu \nu}^{3}$ invariant in the next section. Since the Weyl multiplet consists of gauge fields only, the components of the Weyl multiplet do not show up explicitly in a superconformal Lagrangian. Therefore, alternatively we can start from a rigid higher derivative action for 
a scalar multiplet with appropriate weight, and then consider the conformal supergravity coupled model.

For the 4-derivative case, we need a rigid theory that contains a term proportional to $\mathcal{A} \square \square \mathcal{A}$ since such a term would include the Ricci tensor squared when we couple to conformal gravity and replace this term by $\mathcal{A} \square^{c} \square^{c} \mathcal{A}$. The rigid conformal Lagrangian that includes such a term is given by

$$
\mathcal{L}_{\Phi}=\Phi \square \square \Phi+\frac{1}{16} P \square P,
$$

where $(\Phi, \Psi, P)$ are the fields of a $\omega_{\Phi}=-\frac{1}{2}$ scalar multiplet. Considering the supergravity coupled model, we obtain the following bosonic terms

$$
\begin{aligned}
e^{-1} \mathcal{L}_{\Phi}= & \Phi \square^{c} \square^{c} \Phi+\frac{1}{16} P \square^{c} P \\
= & \left(R_{\mu \nu} R^{\mu \nu}-\frac{23}{64} R^{2}\right) \Phi^{2}+\frac{1}{16} P \square P-\frac{1}{128} R P^{2} \\
& +\frac{3}{2} R \partial_{\mu} \Phi \partial^{\mu} \Phi+\frac{1}{4} R \Phi \square \Phi-4 R_{\mu \nu} \partial^{\mu} \Phi \partial^{\nu} \Phi+(\square \Phi)^{2} .
\end{aligned}
$$

As our supergravity construction is based on a compensating multiplet, we would like to express the $\omega_{\Phi}=-\frac{1}{2}$ multiplet in terms of a compensating multiplet. Using the multiplication rule (2.10), we obtain the following expressions:

$$
\Phi=\phi^{-1}, \quad P=-\phi^{-2} S .
$$

Making this replacement in the Lagrangian (3.15) and imposing the gauge-fixing conditions (2.17), we obtain the supersymmetric completion of the Ricci tensor squared given in (3.13).

\subsection{Supersymetric $R S^{n}$ invariants}

In this subsection, we construct a third supersymmetric invariant which is a supersymmetric extension of the off-diagonal $R S^{2}$ term. In fact, we will construct a whole class of supersymmetric $R S^{n}$ (for any positive integer $n$ ) invariants. This family of invariants was already obtained in [11] using superspace. Here we give an alternative derivation using components. For $n=-1$ the invariant coincides with the supersymmetric cosmological constant (2.28), while for $n=0$ the invariant is given by the supersymmetric Einstein-Hilbert term, see eq. (2.20). For all $n \geq 1$ the invariant does not contain a purely gravitational term.

It is instructive to first construct the $n=2$ invariant and then derive the general formula. Multiplying a neutral multiplet $(\sigma, \psi, N)$ with an auxiliary multiplet $(Z, \Omega, F)$ we obtain another auxiliary multiplet $\left(Z^{\prime}, \Omega^{\prime}, F^{\prime}\right)$ given by

$$
Z^{\prime}=\sigma Z, \quad F^{\prime}=\sigma F+Z N
$$

Substituting these composite expressions into the action formula (2.9) we obtain the following action describing the coupling of a neutral scalar multiplet to an auxiliary scalar multiplet:

$$
e^{-1} \mathcal{L}_{\mathrm{AN}}=\sigma F+Z N
$$


We now substitute the composite expressions given in (2.27) and impose the gauge-fixing conditions (2.17) after which we obtain the following action:

$$
e^{-1} \mathcal{L}_{\mathrm{CN}}=-\frac{1}{2} \sigma S+\frac{1}{16} N .
$$

Finally, applying the map (3.3) in this Lagrangian we obtain

$$
e^{-1} \mathcal{L}_{S^{2}}=R-2 S^{2}
$$

which matches with (the bosonic part of) the Poincaré supergravity Lagrangian (2.20).

It is now straightforward to generalize this construction from $n=2$ to general values of $n$. By sequentially multiplying an auxiliary multiplet with a neutral multiplet, we obtain the general formula

$$
Z^{(n)}=\sigma^{n} Z, \quad F^{(n)}=\sigma^{n} F+n \sigma^{n-1} Z N,
$$

where we have only given the terms relevant to the bosonic part of the $R S^{n}$ action. Using these expressions into the density formula (2.9) we obtain the following bosonic terms

$$
e^{-1} \mathcal{L}_{\mathrm{AN}}^{(n)}=\sigma^{n} F+n \sigma^{n-1} Z N .
$$

Next, upon substituting the composite expressions (2.27), imposing the gauge fixing conditions (2.17) and applying the map (3.3), we obtain the following Lagrangian:

$$
e^{-1} \mathcal{L}_{R S^{n}}=S^{n+2}+\frac{n+1}{6 n-2} R S^{n} .
$$

This Lagrangian agrees with the superspace result given in [11].

In the next section we will need the $n=2$ invariant which is given by

$$
e^{-1} \mathcal{L}_{R S^{2}}=S^{4}+\frac{3}{10} R S^{2} .
$$

For future reference we also give the explicit expression of the $n=4$ invariant:

$$
e^{-1} \mathcal{L}_{R S^{4}}=S^{6}+\frac{5}{22} R S^{4} .
$$

\subsection{Supersymmetric new massive gravity}

Given the results obtained in the previous three subsections we can now write the most general supersymmetric terms of mass dimension 4 as follows:

$$
\begin{aligned}
e^{-1} \mathcal{L}_{\mathrm{SQuad}}= & e^{-1}\left(a_{1} \mathcal{L}_{R_{\mu \nu}^{2}}+a_{2} \mathcal{L}_{R^{2}}+a_{3} \mathcal{L}_{R S^{2}}\right), \\
= & a_{1} R_{\mu \nu} R^{\mu \nu}+a_{2} R^{2}-\left(16 a_{2}+6 a_{1}\right) \partial_{\mu} S \partial^{\mu} S \\
& +\left(4 a_{1}+12 a_{2}+\frac{3}{10} a_{3}\right) R S^{2}+\left(12 a_{1}+36 a_{2}+a_{3}\right) S^{4} .
\end{aligned}
$$

As discussed in the introduction, we would like to cancel the kinetic term for $S$ and the off-diagonal $R S^{2}$ term. This leads to the following two restrictions on the three coefficients $\left(a_{1}, a_{2}, a_{3}\right)$ :

$$
a_{2}=-\frac{3}{8} a_{1}, \quad a_{3}=\frac{5}{3} a_{1}
$$


Therefore, the terms of mass dimension 4 in supersymmetric NMG, or shortly SNMG, are given by

$$
e^{-1} \mathcal{L}_{\mathrm{SNMG}}^{(4)}=R_{\mu \nu} R^{\mu \nu}-\frac{3}{8} R^{2}+\frac{1}{6} S^{4}
$$

This concludes our discussion of how to construct a ghost-free supersymmetric NMG model.

\section{Supersymmetric six-derivative invariants}

In this section we push the analysis of the previous section one level further and consider the $\mathcal{N}=1$ supersymmetrization of the following terms with mass dimension $6:{ }^{6}$

$$
\begin{aligned}
e^{-1} \mathcal{L}^{(6)}= & a_{1} R_{\mu \nu} R^{\nu \rho} R_{\rho}{ }^{\mu}+a_{2} R^{3}+a_{3} R R_{\mu \nu} R^{\mu \nu}+a_{4} R^{2} S^{2}+ \\
& +a_{5} R S^{4}+a_{6} S^{2} R_{\mu \nu} R^{\mu \nu}+a_{7} R_{\mu \nu} \partial^{\mu} S \partial^{\nu} S+ \\
& +a_{8} R \partial_{\mu} S \partial^{\mu} S+a_{9} R S \square S+a_{10} S^{2} \partial_{\mu} S \partial^{\mu} S+a_{11} S^{6}
\end{aligned}
$$

Here $a_{1} \ldots, a_{11}$ are arbitrary coefficients. Requiring supersymmetry will lead to relations between these coefficients leaving us with as many parameters as supersymmetric invariants we construct in this section. As discussed in the introduction, requiring a ghost-free model at the linearized level leads to several restrictions on the remaining independent parameters. These restrictions will be discussed in the next section.

In this section we will merely focus on the construction of the different supersymmetric invariants. In the first subsection we will construct the supersymmetrization of the three purely gravitational terms in the Lagrangian (4.1), corresponding to the coefficients $a_{1}, \ldots, a_{3}$. For completeness, we will also construct the supersymmetric $R \square R$ and $R_{\mu \nu} \square R^{\mu \nu}$ invariants. In the next subsection we will construct the supersymmetric completion of five of the off-diagonal terms in (4.1) corresponding to the coefficients $a_{4}, a_{6}, a_{7}, a_{9}$. The supersymmetric completion of the $a_{5}$-term was already given in eq. (3.25).

\subsection{Diagonal invariants}

In this subsection we will discuss, one after the other, how to obtain the supersymmetric extensions $\mathrm{g} 1, \ldots, \mathrm{g} 5$ of the first three purely gravitational terms in (4.1) [g1, g2, g3], together with the supersymmetric extensions of the $R \square R$ and $R_{\mu \nu} \square R^{\mu \nu}$ terms [g4, g5].

g1: supersymmetric $R^{3}$ invariant. The construction of the supersymmetric $R^{3}$ invariant requires the introduction of a second neutral multiplet $\left(\sigma^{\prime}, \psi^{\prime}, N^{\prime}\right)$. Using the multiplication rule (2.10), we first construct a new auxiliary multiplet $\left(Z^{\prime}, \Omega^{\prime}, F^{\prime}\right)$ in terms of an auxiliary multiplet $(Z, \Omega, F)$ and this second neutral multiplet $\left(\sigma^{\prime}, \psi^{\prime}, N^{\prime}\right)$ :

$$
Z^{\prime}=\sigma^{\prime 2} Z, \quad F^{\prime}=\sigma^{\prime 2} F+2 \sigma^{\prime} Z N^{\prime} .
$$

\footnotetext{
${ }^{6}$ These are the most general terms of mass dimension 6 except for the terms $R_{\mu \nu} \square R^{\mu \nu}, R \square R, S \epsilon_{\mu \nu \rho} R_{\sigma}^{\mu} \nabla^{\rho} R^{\nu \sigma}$ and $(\square S)^{2}$. We do not consider the first two terms since they lead to models with two massive gravitons one of which is a ghost. It turns out that the last two terms are only needed for the supersymmetrization of the first two terms.
} 
We now use the fact the fields of the auxiliary multiplet $(Z, \Omega, F)$ can be expressed in terms of the components of the compensating multiplet as

$$
Z=\phi S, \quad F=16 \phi \square^{c} \phi+S^{2}
$$

by first multiplying a compensating multiplet with a curvature multiplet and, next, substituting the composite expressions (2.14) for the components of the curvature multiplet. Next, we use the action formula for $\left(Z^{\prime}, \Omega^{\prime}, F^{\prime}\right)$ where we write the components as in eq. (4.2) with the substitutions (4.3) being made. This gives rise to the following action that describes the coupling of a neutral multiplet to a compensating multiplet

$$
e^{-1} \mathcal{L}_{\mathrm{CN}}=16 \sigma^{\prime 2} \phi \square \phi-2 R \phi^{2} \sigma^{\prime 2}+S^{2} \sigma^{\prime 2}+2 \sigma^{\prime} \phi S N^{\prime} .
$$

The final step of the construction requires one to express $\sigma^{\prime}$ in terms of the Ricci scalar. To achieve this we make use of the map (3.2) that expresses the components of the neutral scalar multiplet as the product of two compensating multiplets $\left(\phi_{1}, \lambda_{1}, S_{1}\right)$ and $\left(\phi_{2}, \lambda_{2}, S_{2}\right)$. Using the multiplication rule, we can write the components $\left(\phi_{2}, \lambda_{2}, S_{2}\right)$ as

$$
\phi_{2}=\sigma \phi_{1}, \quad \lambda_{2}=\phi_{1} \psi+\sigma \lambda_{1}, \quad S_{2}=\sigma S_{1}+\phi_{1} N+\bar{\lambda}_{1} \psi
$$

Then, using these expressions in (3.2) and setting $\phi_{1}=\phi$, we obtain the following bosonic expressions

$$
\begin{aligned}
\sigma^{\prime} & =\phi^{-2} N+\phi^{-3} \sigma S, \\
N^{\prime} & =16 \phi^{-3} \square^{C}(\phi \sigma)-3 \phi^{-4} \sigma S^{2}-3 \phi^{-3} S N .
\end{aligned}
$$

Next, applying the map (3.2) in the above expressions and gauge fixing according to (2.17), we indeed have expressed $\sigma^{\prime}$ in terms of the Ricci scalar. Finally, substituting the resulting expressions into the action (4.4) we find that the bosonic part of the supersymmetric completion of the $R^{3}$ term is given by

$$
e^{-1} \mathcal{L}_{R^{3}}=R^{3}+14 R^{2} S^{2}+80 R S^{4}+32 R S \square S-384 S^{2} \partial_{\mu} S \partial^{\mu} S+160 S^{6} .
$$

g2: supersymmetric $R R_{\mu \nu} R^{\mu \nu}$ invariant. We next consider the supersymmetric completion of the $R R_{\mu \nu} R^{\mu \nu}$ term. In order to do this we first wish to construct a supersymmetric $\sigma F_{\mu \nu}^{I} F^{\mu \nu I}$ term and next apply the maps (3.3) and (3.7). To achieve this we first multiply the auxiliary multiplet with a neutral multiplet such that we obtain a new auxiliary multiplet. The bosonic composite expressions for the new auxiliary multiplet $\left(Z^{\prime}, \Omega^{\prime}, F^{\prime}\right)$ are given by

$$
Z^{\prime}=\sigma Z, \quad F^{\prime}=\sigma F+Z N .
$$

Plugging this into the action formula (2.9) we obtain the following action:

$$
e^{-1} \mathcal{L}_{\mathrm{AN}}=\sigma F+Z N
$$

Next, we substitute the composite expressions (3.10) to obtain the action

$$
e^{-1} \mathcal{L}_{\sigma F_{\mu \nu}^{2}}=-\frac{1}{4} \sigma F_{\mu \nu}^{I} F^{\mu \nu I},
$$


where we have gauge fixed according to (2.17). Finally, using the composite expressions (4.6), and applying the maps (3.3) and (3.7), we obtain the following supersymmetric invariant:

$$
\begin{aligned}
e^{-1} \mathcal{L}_{R^{3}+R R_{\mu \nu} R^{\mu \nu}}= & R R_{\mu \nu} R^{\mu \nu}-\frac{1}{4} R^{3}-2 R \partial_{\mu} S \partial^{\mu} S+4 S^{2} R_{\mu \nu} R^{\mu \nu}+7 R S^{4} \\
& -8 S^{2} \partial_{\mu} S \partial^{\mu} S+12 S^{6}
\end{aligned}
$$

Combining this action with the supersymmetric $R^{3}$ action, see eq. (4.7), we find that the supersymmetric completion of the $R R_{\mu \nu} R^{\mu \nu}$ invariant is given by:

$$
\begin{aligned}
e^{-1} \mathcal{L}_{R R_{\mu \nu} R^{\mu \nu}}= & R R_{\mu \nu} R^{\mu \nu}+4 S^{2} R_{\mu \nu} R^{\mu \nu}-2 R \partial_{\mu} S \partial^{\mu} S+8 R S \square S \\
& +\frac{7}{2} R^{2} S^{2}+27 R S^{4}-104 S^{2} \partial_{\mu} S \partial^{\mu} S+52 S^{6} .
\end{aligned}
$$

g3: supersymmetric $R_{\mu \nu}^{3}$ invariant. We now discuss the construction of the supersymmetric Ricci tensor cube invariant. Unlike the other invariants, the $R_{\mu \nu}^{3}$ cannot cannot be obtained by applying a map between a scalar or Yang-Mills multiplet and the Weyl multiplet. Instead, we look for a K-invariant conformal quantity that gives rise to a $R_{\mu \nu}^{3}$ action. Given the following K-transformations

$$
\begin{aligned}
\delta_{K} \square^{c} \mathcal{A} & =-2\left(2 \omega_{\mathcal{A}}-1\right) \Lambda_{K}^{a} \mathcal{D}_{a} \mathcal{A}, \\
\delta_{K} \square^{c} \square^{c} \mathcal{A} & =-4\left(2 \omega_{\mathcal{A}}+1\right) \Lambda_{K}^{a} \mathcal{D}_{a} \square^{c} \mathcal{A},
\end{aligned}
$$

we observe that the $\phi \square^{c} \phi$ and $\Phi \square^{c} \square^{c} \Phi$ terms, which gave rise to a supersymmetric Poincaré and $R_{\mu \nu}^{2}$ action arose naturally by demanding $\mathrm{K}$-invariance and by requiring the conformal weight to match the required factor +3 . A natural generalization of this construction is to consider

$$
\delta_{K} \square^{c} \square^{c} \square^{c} \mathcal{A}=-6\left(2 \omega_{\mathcal{A}}+3\right) \Lambda_{K}^{a} \mathcal{D}_{a} \square^{c} \square^{c} \mathcal{A} .
$$

This transformation rule shows that given a scalar multiplet $(\tau, \zeta, L)$ with the conformal weight of the lowest component given by $\omega_{\tau}=-\frac{3}{2}$, the following Lagrangian gives rise to a supersymmetric completion of the $R_{\mu \nu}^{3}$ term:

$$
\begin{aligned}
e^{-1} \mathcal{L}_{\tau}= & \tau \square^{c} \square^{c} \square^{c} \tau+\frac{1}{16} L \square^{c} \square^{c} L \\
= & \left(\frac{3}{2} R_{\mu \nu} \partial^{\mu} \partial^{\nu} R-\frac{39}{64} R \square R+24 R_{\mu \nu} R^{\nu \rho} R_{\rho}{ }^{\mu}-\frac{45}{2} R R_{\mu \nu} R^{\mu \nu}+\frac{2463}{512} R^{3}\right) \tau^{2} \\
& +\frac{1}{16}\left(R_{\mu \nu} R^{\mu \nu}-\frac{23}{32} R^{2}\right) L^{2}+\frac{3}{32} R \partial_{\mu} L \partial^{\mu} L+\frac{1}{64} R L \square L \\
& -\frac{1}{4} R_{\mu \nu} \partial^{\mu} L \partial^{\nu} L+\frac{1}{16}(\square L)^{2} .
\end{aligned}
$$

Since our supergravity construction is based on a compensating multiplet, we would like to express the $\omega_{\tau}=-\frac{3}{2}$ multiplet in terms of the compensating multiplet. Using the multiplication rule (2.10), we obtain the following expressions:

$$
\tau=\phi^{-3}, \quad L=-3 \phi^{-4} S
$$


Making this replacement in the above action and gauge fixing according to (2.17), we obtain the following supersymmetric completion of the Ricci tensor cube term:

$$
\begin{aligned}
e^{-1} \mathcal{L}_{R_{\mu \nu}^{3}}^{\prime}= & \frac{3}{64} R \square R+8 R_{\mu \nu} R^{\nu \rho} R_{\rho}{ }^{\mu}-\frac{15}{2} R R_{\mu \nu} R^{\mu \nu}+\frac{821}{512} R^{3} \\
& +\frac{3}{4} R_{\mu \nu} R^{\mu \nu} S^{2}-\frac{69}{256} R^{2} S^{2}+\frac{9}{8} R \partial_{\mu} S \partial^{\mu} S-3 R^{\mu \nu} \partial_{\mu} S \partial_{\nu} S \\
& +\frac{3}{16} R S \square S+\frac{3}{4}(\square S)^{2} .
\end{aligned}
$$

Subtracting the $R^{3}, R R_{\mu \nu} R^{\mu \nu}$ invariants and the $R \square R$ invariant, which we shall construct below, see eq. (4.19), the final expression of the $R_{\mu \nu}^{3}$ action reads

$$
\begin{aligned}
e^{-1} \mathcal{L}_{R_{\mu \nu}^{3}}= & R^{\mu \nu} R_{\mu}{ }^{\rho} R_{\nu \rho}+\frac{123}{32} S^{2} R^{\mu \nu} R_{\mu \nu}+\frac{223}{512} R^{2} S^{2}-\frac{117}{64} R \partial^{\mu} S \partial_{\mu} S \\
& +\frac{31}{32} R S \square S-\frac{3}{8} R^{\mu \nu} \partial_{\mu} S \partial_{\nu} S-\frac{309}{16} S^{2} \partial^{\mu} S \partial_{\mu} S+\frac{2357}{256} R S^{4} \\
& +\frac{527}{32} S^{6} .
\end{aligned}
$$

g4: supersymmetric $R \square R$ invariant. To construct the $R_{\mu \nu}^{3}$ invariant above we needed to know the result for the $R \square R$ invariant. We will derive this expression here. To construct the supersymmetric completion of a $R \square R$ term we make use of the following observation. When we constructed the supersymmetric $R^{2}$ invariant in section 3.1, we started from a two-derivative action for a neutral scalar multiplet. Next, after gauge fixing the superconformal symmetries, we obtained the supersymmetric $R^{2}$ invariant by making use of the map (3.3) between the neutral scalar multiplet and the Weyl multiplet in which the auxiliary field of the neutral scalar multiplet, $N$, is mapped to the torsionful Ricci scalar. This suggests that, to obtain a supersymmetric $R \square R$ term, we should start from a four derivative action for the same scalar multiplet such that the auxiliary scalar $N$ occurs as a $N \square N$ term. Under the map (3.3), this term will give rise to the desired $R \square R$ term.

The problem of constructing a $R \square R$ invariant is now reduced to constructing a fourderivative action for a neutral scalar multiplet. Such an action can easily be constructed by using the composite expressions (4.6) into the action for the neutral scalar multiplet. Gauge fixing via (2.17) and using the gauge fixed expressions for (4.6) into the action (3.5) we obtain the following supersymmetric completion of the $R \square R$ term:

$$
\begin{aligned}
e^{-1} \mathcal{L}_{R \square R}= & R \square R+24 R S \square S+16 R \partial_{\mu} S \partial^{\mu} S-208 S^{2} \partial_{\mu} S \partial^{\mu} S+16(\square S)^{2} \\
& +R^{2} S^{2}+12 R S^{4}+36 S^{6} .
\end{aligned}
$$

g5: supersymmetric $R_{\mu \nu} \square R^{\mu \nu}$ invariant. For completeness, we construct the supersymmetric completion of a $R_{\mu \nu} \square R^{\mu \nu}$ term. In the same way that we used in subsection 3.2 the gauge-fixed supersymmetric $F_{\mu \nu} F^{\mu \nu}$ action and the map (3.7) to construct a supersymmetric $R_{\mu \nu} R^{\mu \nu}$ invariant we expect that a supersymmetric $R_{\mu \nu} \square R^{\mu \nu}$ action can be constructed from applying (3.7) to a supersymmetric $F_{\mu \nu} \square F^{\mu \nu}$ action. Our main task is to construct such an action.

Our starting point is the gauge-fixed supersymmetric Yang-Mills action (3.11) with gauge vector $C_{\mu}^{I}$ and curvature $G_{\mu \nu}^{I}$. We observe that upon writing

$$
G_{\mu \nu}^{I}=\epsilon_{\mu \nu \rho} \nabla_{\sigma} F^{\rho \sigma I}
$$


the leading $G_{\mu \nu}^{I} G^{\mu \nu I}$ term gets mapped to a higher-derivative $F_{\mu \nu}^{I} \square F^{\mu \nu I}$ term. Therefore, using (4.20), we wish to map a conformal Yang-Mills multiplet $\left(A_{\mu}^{I}, \varphi^{I}\right)$ to another conformal Yang-Mills multiplet $\left(C_{\mu}^{I}, \Omega^{I}\right)$. We find that this is achieved by the following map:

$$
\begin{aligned}
\Omega^{I} & =\phi^{-2} \not \mathcal{D} \varphi^{I}+\frac{1}{8} \phi^{-3} \gamma \cdot \widehat{F}^{I} \lambda-\phi^{-3} \not \mathcal{D} \phi \varphi^{I}+\frac{1}{4} \phi^{-3} S \varphi^{I}-\frac{3}{8} \phi^{-4} \varphi^{I} \bar{\lambda} \lambda \\
\widehat{G}_{\mu \nu}^{I} & =\epsilon_{\mu \nu \rho} \mathcal{D}_{\sigma}\left(\phi^{-2} \widehat{F}^{\rho \sigma I}\right) .
\end{aligned}
$$

Applying this map we find that, after gauge-fixing, the supersymmetric completion of a $F_{\mu \nu} \square F^{\mu \nu}$ term does not require any other bosonic term:

$$
e^{-1} \mathcal{L}_{F_{\mu \nu} \square F^{\mu \nu}}=\nabla_{\mu} F^{\mu \nu I} \nabla^{\rho} F_{\nu \rho}^{I}
$$

Next, applying the map (3.7) between a Yang-Mills multiplet and the Weyl multiplet, we obtain the following action

$$
\begin{aligned}
e^{-1} \mathcal{L}_{R_{\mu \nu} \square R^{\mu \nu}}^{\prime}= & R_{\mu \nu} \square R^{\mu \nu}-\frac{1}{4} R \square R-3 R_{\mu \nu} R^{\nu \rho} R_{\rho}{ }^{\mu}+\frac{5}{2} R R_{\mu \nu} R^{\mu \nu} \\
& -\frac{1}{2} R^{3}-14 R S \square S-14 R \partial_{\mu} S \partial^{\mu} S-16 \epsilon^{\mu \rho \sigma} S R_{\mu}{ }^{\nu} \nabla_{\rho} R_{\nu \sigma} \\
& +16 R_{\mu \nu} \partial^{\mu} S \partial^{\nu} S+2(\square S)^{2}+24 S^{2} \partial_{\mu} S \partial^{\mu} S \\
& -16 R^{2} S^{2}+64 S^{2} R_{\mu \nu} R^{\mu \nu}+64 R S^{4}+192 S^{6} .
\end{aligned}
$$

Finally, subtracting the $R_{\mu \nu}^{3}, R R_{\mu \nu} R^{\mu \nu}, R^{3}$ and $R \square R$ invariants, we obtain the desired supersymmetric completion of the $R_{\mu \nu} \square R^{\mu \nu}$ invariant

$$
\begin{aligned}
e^{-1} \mathcal{L}_{R_{\mu \nu} \square R^{\mu \nu}}= & R_{\mu \nu} \square R^{\mu \nu}+\frac{2097}{32} S^{2} R_{\mu \nu} R^{\mu \nu}-\frac{8291}{512} R^{2} S^{2}+\frac{17183}{256} R S^{4} \\
& -\frac{671}{64} R \partial_{\mu} S \partial^{\mu} S-\frac{291}{32} R S \square S+\frac{119}{8} R_{\mu \nu} \partial^{\mu} S \partial^{\nu} S \\
& -16 \epsilon^{\mu \rho \sigma} S R_{\mu}{ }^{\nu} \nabla_{\rho} R_{\nu \sigma}-\frac{287}{16} S^{2} \partial_{\mu} S \partial^{\mu} S+6(\square S)^{2}+\frac{6413}{32} S^{6} .
\end{aligned}
$$

\subsection{Off-diagonal invariants}

In this subsection we will construct, one after the other, the supersymmetric invariants o1 $\ldots, \mathrm{o} 4$, corresponding to the off-diagonal $a_{5}, a_{6}, a_{7}, a_{9}$ terms in (4.1). For completeness, we will also give the supersymmetric extension of the off-diagonal term $\epsilon^{\mu \nu \rho} S R_{\mu}{ }^{\sigma} \nabla_{\nu} R_{\sigma \rho}$ which will not be needed since it only occurs in the $R_{\mu \nu} \square R^{\mu \nu}$ invariant. The 5th off-diagonal invariant that will be needed is the supersymmetric completion of the $R S^{4}$ term which was already given in eq. (3.25).

o1: supersymmetric $S^{2 n} R^{2}$ invariants. In the previous subsection we have shown that the supersymmetric cosmological constant and Poincaré supergravity are special cases of a more general supersymmetric $R S^{n}$ invariant for $n=-1$ and $n=0$. Here we will show that similarly the $R^{2}$ Lagrangian (3.6) can be seen as a special case of a more general supersymmetric $S^{2 n} R^{2}$ invariant for $n=0$. In order to do so, it is instructive to first re-consider the $n=0$ case. We start from the superconformal scalar multiplet Lagrangian (2.16) for 
a compensating scalar multiplet. Next, using the multiplication rule (2.10), we replace $\phi$ with $\sigma \phi$ and $S$ with $(\sigma S+\phi N)$. This leads to the following Lagrangian:

$$
e^{-1} \mathcal{L}_{\mathrm{SC}}=16(\sigma \phi) \square(\sigma \phi)-2 R \phi^{2} \sigma^{2}+(\sigma S+\phi N)^{2}
$$

Substituting the superconformal gauge fixing conditions (2.17) and applying (3.3) we obtain

$$
e^{-1} \mathcal{L}_{R^{2}}^{\prime}=4 S \square S+4 S^{2}+\frac{3}{2} R S^{2}+\frac{1}{4} R^{2}
$$

Combining this Lagrangian with the $S^{4}$ action given by (3.23) for $n=3$ we recover the $R^{2}$ Lagrangian (3.6) in the form we derived earlier:

$$
e^{-1} \mathcal{L}_{R^{2}}=e^{-1}\left(\mathcal{L}_{R^{2}}^{\prime}+5 \mathcal{L}_{S^{4}}\right)=4 S \square S+9 S^{4}+3 R S^{2}+\frac{1}{4} R^{2}
$$

We next consider the general case and take a compensating scalar multiplet coupled to $n$ copies of a neutral scalar multiplet. In that case we replace in the superconformal scalar multiplet Lagrangian (2.16) $\phi$ with $\sigma^{n} \phi$ and $S$ with $\sigma^{n} S+n \sigma^{n-1} \phi N$ and obtain:

$$
e^{-1} \mathcal{L}_{\mathrm{CN}^{n}}=16\left(\sigma^{n} \phi\right) \square\left(\sigma^{n} \phi\right)-2 R \sigma^{2 n} \phi^{2}+\left(\sigma^{n} S+n \sigma^{n-1} \phi N\right)^{2} .
$$

Finally, substituting the superconformal gauge-fixing conditions (2.17) and applying (3.3) we obtain

$$
\begin{aligned}
e^{-1} \mathcal{L}_{S^{2 n} R^{2}}= & -4(n+1)^{2} S^{2 n} \partial_{\mu} S \partial^{\mu} S+\frac{1}{4}(n+1)^{2} R^{2} S^{2 n}+(3 n+2)^{2} S^{2 n+4} \\
& +\left(3 n^{2}+5 n+\frac{3}{2}\right) R S^{2 n+2}
\end{aligned}
$$

which reduces for $n=0$ to the supersymmetric invariant given in (4.26). For simplicity, in the off-diagonal case, we will use a basis where terms that belong to other off-diagonal invariants, like the last term in the above Lagrangian, have not been canceled. We will only do this for the purely gravitational terms.

o2: supersymmetric $S^{n} R_{\mu \nu} R^{\mu \nu}$ invariants. The Ricci tensor squared Lagrangian (3.12) can also be obtained as a special case of a more general supersymmetric $S^{n} R_{\mu \nu}^{2}$ Lagrangian by setting $n=0$. The construction is based on the superconformal action (3.22). Substituting the composite expressions (3.10) into (3.22) and gauge-fixing according to (2.17) we obtain the following Lagrangian

$$
e^{-1} \mathcal{L}_{\sigma^{n} F_{\mu \nu}^{2}}=-\frac{1}{4} \sigma^{n} F_{\mu \nu}^{I} F^{\mu \nu I}
$$

Next, after applying (3.3) and (3.7) we obtain the Lagrangian:

$$
e^{-1} \mathcal{L}_{S^{n}} R_{\mu \nu}^{2}=-S^{n} R_{\mu \nu} R^{\mu \nu}+\frac{1}{4} S^{n} R^{2}+2 S^{n} \partial_{\mu} S \partial^{\mu} S-R S^{n+2}-3 S^{n+4} .
$$


o3: supersymmetric $R_{\mu \nu} \partial^{\mu} S \partial^{\nu} S$ invariant. The construction of the $R_{\mu \nu} \partial^{\mu} S \partial^{\nu} S$ invariant is based on the superconformal action (3.15). Using the multiplication rule, we replace the $\omega_{\Phi}=-\frac{1}{2}$ multiplet with another $\omega_{\Phi}=-\frac{1}{2}$, multiplet, i.e., $\Phi \rightarrow \sigma \Phi$ and $P \rightarrow \sigma P+\Phi N$. After gauge fixing, the resulting action reads

$$
\begin{aligned}
e^{-1} \mathcal{L}_{R_{\mu \nu} \partial^{\mu} S \partial^{\nu} S}^{\prime}= & 4 R_{\mu \nu} R^{\mu \nu} S^{2}-\frac{31}{16} R^{2} S^{2}-64 S^{2} \partial_{\mu} S \partial^{\mu} S+9 R S \square S+14 R \partial_{\mu} S \partial^{\mu} S \\
& +\frac{1}{4} R \square R-2 R S^{4}-\frac{1}{32} R^{3}-16 R_{\mu \nu} \partial^{\mu} S \partial^{\nu} S+4(\square S)^{2} .
\end{aligned}
$$

Subtracting the $R^{3}$ and $R \square R$ invariants, we find that the $R_{\mu \nu} \partial^{\mu} S \partial^{\nu} S$ invariant is given by

$$
\begin{aligned}
e^{-1} \mathcal{L}_{R_{\mu \nu} \partial^{\mu} S \partial^{\nu} S}= & R_{\mu \nu} \partial^{\mu} S \partial^{\nu} S-\frac{5}{8} R \partial_{\mu} S \partial^{\mu} S-\frac{1}{4} R S \square S-\frac{1}{4} S^{2} R_{\mu \nu} R^{\mu \nu} \\
& +\frac{7}{64} R^{2} S^{2}+\frac{5}{32} R S^{4}+\frac{3}{2} S^{2} \partial_{\mu} S \partial^{\mu} S+\frac{1}{4} S^{6} .
\end{aligned}
$$

o4: supersymmetric $R S \square S$ invariant. For the construction of the supersymmetric $R S \square S$ invariant, our starting point is the action for a neutral scalar multiplet $\left(\sigma^{\prime}, \psi^{\prime}, N^{\prime}\right)$ coupled to an auxiliary scalar multiplet $(Z, \Omega, F)$, see eq. (3.18):

$$
e^{-1} \mathcal{L}_{\mathrm{AN}}=\sigma^{\prime} F+Z N^{\prime}
$$

Following the same strategy that was used when we constructed the Ricci scalar squared invariant, this action can be cast into the following form:

$$
e^{-1} \mathcal{L}_{N^{2}}=\sigma^{\prime}\left(\partial_{\mu} \sigma \partial^{\mu} \sigma-\frac{1}{16} N^{2}\right)
$$

Next, using the maps (3.3) and (4.6), we obtain the following $R^{3}$ action

$$
e^{-1} \mathcal{L}_{R^{3}}^{\prime}=R^{3}+16 R^{2} S^{2}+84 R S^{4}-16 R \partial_{\mu} S \partial^{\mu} S-64 S^{2} \partial_{\mu} S \partial^{\mu} S+144 S^{6} .
$$

Finally, removing the $R^{3}$ invariant found in (4.7), we find that the $R S \square S$ invariant is given by

$$
e^{-1} \mathcal{L}_{R S \square S}=R S \square S+\frac{1}{2} R \partial_{\mu} S \partial^{\mu} S-\frac{1}{16} R^{2} S^{2}-\frac{1}{8} R S^{4}-10 S^{2} \partial_{\mu} S \partial^{\mu} S+\frac{1}{2} S^{6} .
$$

o5: supersymmetric $S^{n} \epsilon_{\mu \sigma \lambda} R^{\mu}{ }_{\nu} \nabla^{\sigma} R^{\lambda \nu}$ invariant. In this section, we consider the supersymmetrization of the $S^{n} \epsilon_{\mu \sigma \lambda} R^{\mu}{ }_{\nu} \nabla^{\sigma} R^{\lambda \nu}$ term. The $n=0$ case corresponds to the purely gravitational parity-odd term $\epsilon_{\mu \nu \rho} R^{\mu}{ }_{\sigma} \nabla^{\nu} R^{\rho \sigma}$. Our starting point for the construction procedure is the Lagrangian that describes the coupling of two Yang-Mills multiplets to $n$ number of neutral multiplets

$$
e^{-1} \mathcal{L}_{2 Y M}=-\frac{1}{4} \sigma^{n} F_{\mu \nu}^{I} G^{\mu \nu I}
$$

where the curvature $G_{\mu \nu}^{I}$ is the curvature of the gauge vector $C_{\mu}^{I}$. We observe that once again, upon writing $G_{\mu \nu}^{I}=\epsilon_{\mu \nu \rho} \nabla_{\sigma} F^{\rho \sigma I}$, the leading $G_{\mu \nu}^{I} F^{\mu \nu I}$ term gets mapped to a higher-derivative $\epsilon_{\mu \nu \rho} F^{\mu}{ }_{\sigma}^{I} \nabla_{\nu} F^{\rho \sigma I}$ term, which leads to the desired supersymmetric higher derivative action via the Yang-Mills to Poincaré multiplet map. Therefore, using (4.21) we obtain the following supersymmetric completion of a $\sigma^{n} \epsilon_{\mu \nu \rho} F^{\mu}{ }_{\sigma}^{I} \nabla_{\nu} F^{\rho \sigma I}$ term:

$$
e^{-1} \mathcal{L}_{\sigma^{n} \epsilon_{\mu \nu \rho} F^{\mu} \sigma_{\sigma}^{I} \nabla^{\nu} F^{\rho \sigma I}}=\sigma^{n} \epsilon_{\mu \nu \rho} F_{\sigma}^{\mu I} \nabla^{\nu} F^{\rho \sigma I} .
$$


Finally, using the neutral multiplet to Poincaré map (3.3) and the Yang-Mills to Poincaré multiplet map (3.7), we obtain

$$
\begin{aligned}
e^{-1} \mathcal{L}_{S^{n} \epsilon_{\mu \nu \rho} R^{\mu} \sigma \nabla^{\nu} R^{\rho \sigma}}= & S^{n} \epsilon_{\mu \nu \rho} R_{\sigma}^{\mu} \nabla^{\nu} R^{\rho \sigma}-n S^{n-1} R_{\mu \nu} \partial^{\mu} S \partial^{\nu} S+n S^{n-1} R \partial_{\mu} S \partial^{\mu} S \\
& +R S^{n} \square S-2(n+12) S^{n+1} \partial_{\mu} S \partial^{\mu} S+8 S^{n+1} R_{\mu \nu} R^{\mu \nu} \\
& -2 R^{2} S^{n+1}+8 R S^{n+3}+24 S^{n+5}
\end{aligned}
$$

\section{Towards 3D Born-Infeld supergravity}

In this section, we use the results we obtained in the previous section to construct a supersymmetric ghost-free higher-derivative gravity model. First, in the next subsection we construct the supersymmetric cubic extended NMG model. Next, in a separate subsection, we use this result to propose an expression for the bosonic part of the supersymmetric 3D Born-Infeld gravity model. In particular, we will show that different truncations of a perturbative expansion of this expression gives rise to the supersymmetric NMG model and the supersymmetric cubic extended NMG model.

\subsection{Supersymmetric cubic extended NMG}

Given the supersymmetric invariants we constructed in the previous section we are now in a position to calculate the $\mathcal{N}=1$ supersymmetric completion of cubic extended NMG. To avoid ghostlike massive gravitons, we restrict ourselves to the use of curvature terms without derivatives, hence we do not consider $R \square R$ and $R_{\mu \nu} \square R^{\mu \nu}$ terms. In this case, the most general supersymmetric invariant of mass dimension 6 is given by

$$
\begin{aligned}
e^{-1} \mathcal{L}^{(6)}= & e^{-1}\left(a_{1} \mathcal{L}_{R_{\mu \nu}^{3}}+a_{2} \mathcal{L}_{R R_{\mu \nu} R^{\mu \nu}}+a_{3} \mathcal{L}_{R^{3}}+a_{4} \mathcal{L}_{R^{2} S^{2}}+a_{5} \mathcal{L}_{S^{2} R_{\mu \nu}^{2}}\right. \\
& \left.+a_{6} \mathcal{L}_{R_{\mu \nu} \partial^{\mu} S \partial^{\nu} S}+a_{7} \mathcal{L}_{R S \square S}+a_{8} \mathcal{L}_{R S^{4}}\right) \\
= & a_{1} R_{\mu \nu} R^{\nu \rho} R_{\rho}{ }^{\mu}+a_{2} R R_{\mu \nu} R^{\mu \nu}+a_{3} R^{3} \\
& +\left(-\frac{3}{8} a_{1}+a_{6}\right) R_{\mu \nu} \partial^{\mu} S \partial^{\nu} S+\left(\frac{31}{32} a_{1}+8 a_{2}+32 a_{3}-\frac{1}{4} a_{6}+a_{7}\right) R S \square S \\
& +\left(\frac{123}{32} a_{1}+4 a_{2}-a_{5}-\frac{1}{4} a_{6}\right) S^{2} R_{\mu \nu} R^{\mu \nu}+\left(-\frac{117}{64} a_{1}-2 a_{2}-\frac{5}{8} a_{6}+\frac{1}{2} a_{7}\right) R \partial_{\mu} S \partial^{\mu} S \\
& +\left(\frac{223}{512} a_{1}+\frac{7}{2} a_{2}+14 a_{3}+a_{4}+\frac{1}{4} a_{5}+\frac{7}{64} a_{6}-\frac{1}{16} a_{7}\right) R^{2} S^{2} \\
& +\left(-\frac{309}{16} a_{1}-104 a_{2}-384 a_{3}-16 a_{4}+2 a_{5}+\frac{3}{2} a_{6}-10 a_{7}\right) S^{2} \partial_{\mu} S \partial^{\mu} S \\
& +\left(\frac{2357}{256} a_{1}+27 a_{2}+80 a_{3}+\frac{19}{2} a_{4}-a_{5}+\frac{5}{32} a_{6}-\frac{1}{8} a_{7}+\frac{5}{22} a_{8}\right) R S^{4} \\
& +\left(\frac{527}{32} a_{1}+52 a_{2}+160 a_{3}+25 a_{4}-3 a_{5}+\frac{1}{4} a_{6}+\frac{1}{2} a_{7}+a_{8}\right) S^{6} .
\end{aligned}
$$

corresponding to the 8 supersymmetric invariants we have constructed. The requirement that all off-diagonal terms and the term $S^{2} \partial_{\mu} S \partial^{\mu} S$ should vanish to avoid ghosts when linearizing around an $\mathrm{AdS}_{3}$ background leads to 7 relations between the 8 coefficients. Setting $a_{1}=1$ we find the following unique solution:

$$
a_{2}=-\frac{9}{8}, \quad a_{3}=\frac{17}{64}, \quad a_{4}=-\frac{3}{32}, \quad a_{5}=-\frac{3}{4}, \quad a_{6}=\frac{3}{8}, \quad a_{7}=-\frac{3}{8}, \quad a_{8}=-\frac{33}{160} .
$$


Substituting these values into the action (5.1), we find that the terms of mass dimension 6 in the supersymmetric cubic extended new massive gravity model, shortly called the SCNMG model, are given by

$$
e^{-1} \mathcal{L}_{\mathrm{SCNMG}}^{(6)}=R_{\mu \nu} R^{\nu \rho} R_{\rho}{ }^{\mu}-\frac{9}{8} R R_{\mu \nu} R^{\mu \nu}+\frac{17}{64} R^{3}+\frac{3}{40} S^{6} .
$$

The fact that supersymmetry and the absence of ghosts at the linearized level uniquely leads to the expression (5.3) is one of the main results of this work.

\subsection{Towards 3D Born-Infeld supergravity}

An example of a specific 3D higher-derivative gravity theory with an infinite number of higher-derivative terms is the Born-Infeld gravity theory

$$
I_{\mathrm{BI}}\left(G_{\mu \nu}\right)=4 m^{2} \int d^{3} x \sqrt{-g}-4 m^{2} \int d^{3} x \sqrt{-\operatorname{det}\left(g_{\mu \nu}-\frac{1}{m^{2}} G_{\mu \nu}\right)},
$$

where $G_{\mu \nu}$ is the Einstein tensor and $m$ is a mass parameter. It was observed in [6] that upon making a perturbative expansion in $1 / m$ and keeping only terms linear and quadratic in the Einstein tensor one ends up with the ghost-free NMG model [4]. Furthermore, keeping also the terms of mass dimension six in the Einstein tensor one ends up with the six-derivative cubic extended NMG model $[6,8]$. The non-trival thing is that these models are reproduced by a minimal BI model, i.e. one which has only terms linear in the Einstein tensor in the determinant. An expansion up to terms of mass dimension 8 has also been considered in [18]. To be explicit, the expansion of the Born-Infeld gravity theory (5.4) including the mass dimension eight terms is given by

$$
\begin{aligned}
e^{-1} \mathcal{L}_{\mathrm{BI}}^{(8)}= & -R+\frac{1}{m^{2}}\left(R_{\mu \nu} R^{\mu \nu}-\frac{3}{8} R^{2}\right)+\frac{2}{3 m^{4}}\left(R_{\mu \nu} R^{\nu \rho} R_{\rho}{ }^{\mu}-\frac{9}{8} R R_{\mu \nu} R^{\mu \nu}+\frac{17}{64} R^{3}\right) \\
-\frac{1}{8 m^{6}}[ & R_{\mu \nu} R^{\nu \rho} R_{\rho \sigma} R^{\sigma \mu}-\frac{5}{3} R R_{\mu \nu} R^{\nu \rho} R_{\rho}{ }^{\mu}+\frac{19}{16} R^{2} R_{\mu \nu} R^{\mu \nu} \\
& \left.\quad-\frac{1}{4}\left(R_{\mu \nu} R^{\mu \nu}\right)^{2}-\frac{169}{768} R^{4}\right] .
\end{aligned}
$$

Comparing the above expansion of the Born-Infeld gravity theory with the results of this work, it is interesting to speculate about the bosonic part of the $\mathcal{N}=1$ Born-Infeld supergravity theory, in particular about its dependence on the auxiliary scalar. Requiring supersymmetry in lowest orders of a perturbative expansion leads us to consider the following expression (without fermions and no cosmological constant)

$$
\begin{aligned}
I_{\mathrm{SBI}}\left(G_{\mu \nu}, S\right)= & +4 m^{2} \int d^{3} x \sqrt{-g}\left(1+\frac{2}{m^{2}} S^{2}\right)- \\
& -4 m^{2} \int d^{3} x \sqrt{-\operatorname{det}\left(g_{\mu \nu}-\frac{1}{m^{2}}\left(G_{\mu \nu}-g_{\mu \nu} S^{2}\right)\right)} .
\end{aligned}
$$


The perturbative expansion of this expression up to terms of mass dimension 8 is given by

$$
\begin{aligned}
& e^{-1} \mathcal{L}_{\mathrm{SBI}}^{(8)}=-(R+ \\
&+\frac{2}{3 m^{4}}\left(S^{2}\right)+\frac{1}{m^{2}}\left(R_{\mu \nu} R^{\nu \rho} R_{\rho}{ }^{\mu}-\frac{9}{8} R R_{\mu \nu} R^{\mu \nu}+\frac{3}{8} R^{2}-\frac{1}{2} R S^{2}-\frac{3}{2} S^{4}-\frac{3}{4} S^{2} R_{\mu \nu} R^{\mu \nu}\right. \\
&\left.\quad+\frac{3}{16} R S^{4}+\frac{9}{32} R^{2} S^{6}+\frac{3}{8} S^{6}\right) \\
&-\frac{1}{8 m^{6}}\left(R_{\mu \nu} R^{\nu \rho} R_{\rho \sigma} R^{\sigma \mu}-\frac{5}{3} R R_{\mu \nu} R^{\nu \rho} R_{\rho}{ }^{\mu}+\frac{19}{16} R^{2} R_{\mu \nu} R^{\mu \nu}\right. \\
& \quad-\frac{1}{4}\left(R_{\mu \nu} R^{\mu \nu}\right)^{2}-\frac{169}{768} R^{4}-2 R_{\mu \nu} R^{\nu \rho} R_{\rho}{ }^{\mu} S^{2}+\frac{9}{4} R R_{\mu \nu} R^{\mu \nu} S^{2} \\
&\left.+\frac{3}{4} R_{\mu \nu} R^{\mu \nu} S^{4}-\frac{17}{32} R^{3} S^{2}-\frac{1}{8} R S^{6}-\frac{9}{32} R^{2} S^{4}-\frac{3}{16} S^{6}\right) .
\end{aligned}
$$

The above Lagrangian has many off-diagonal terms. Without a cosmological constant these terms are harmless. The cosmological extension of (5.6) can easily be obtained by adding the off-shell cosmological constant (2.28). Now the off-diagonal terms are un-acceptable and they need to be canceled by subtracting from the above expression a number of off-diagonal invariants. The subtraction of these off-diagonal invariants not only cancel the off-diagonal terms, they also change the coefficient in front of the purely auxiliary terms. We have verified that the above expression (5.6), after adding a cosmological constant and canceling all off-diagonal terms for the terms up to mass dimension 6 precisely yields the bosonic part of (i) $3 \mathrm{D} \mathcal{N}=1$ Einstein supergravity; (ii) supersymmetric NMG and (iii) supersymmetric cubic extended NMG. In each case the purely gravitational and auxiliary part of these theories is reproduced. What happens if we include the terms of mass dimension 8 is not yet known at this point. However, upon counting the number of off-diagonal terms and the number of possible off-diagonal invariants, both of mass dimension 8 , we find that we have enough invariants to cancel all the off-diagonal terms in the above eight derivative model.

In view of the above we propose that the bosonic part of the $\mathcal{N}=1 \mathrm{BI}$ supergravity model is given by $I_{\mathrm{SBI}}^{\prime}\left(G_{\mu \nu}, S, M\right)$ where $I_{\mathrm{SBI}}\left(G_{\mu \nu}, S, M\right)$ indicates the cosmological extension, with a new cosmological mass parameter $M$, of $I_{\mathrm{SBI}}\left(G_{\mu \nu}, S\right)$, given in eq. (5.6), and where the prime indicates that after expanding the expression of $I_{\mathrm{SBI}}\left(G_{\mu \nu}, S, M\right)$ one cancels all off-diagonal terms by adding a number of off-diagonal invariants to the expression for $I_{\mathrm{SBI}}\left(G_{\mu \nu}, S, M\right)$.

As it stands, the expression for our proposal $I_{\mathrm{SBI}}^{\prime}\left(G_{\mu \nu}, S, M\right)$ is not well-defined to all orders in an expansion of $1 / \mathrm{m}$. The reason for this is that one first has to know the expressions for all off-diagonal supersymmetric invariants that need to be subtracted in order to obtain a ghost-free model around the supersymmetric $A d S_{3}$ vacua. As mentioned above these supersymmetric invariants will contain purely auxiliary terms with powers of $S$ that will modify the coefficients of similar terms that arise from the perturbative expansion of the Lagrangian (5.6). Therefore, without the knowledge of all off-diagonal invariants, we cannot predict the final coefficients of the purely auxiliary terms. We only verified our proposal up to terms of mass dimension 6 . To improve on this one should presumably work with a manifestly supersymmetric formulation using superfields. 


\section{Conclusions}

In this paper we constructed the most general $\mathcal{N}=1$ higher-derivative supergravity action containing terms with no more than 6 derivatives. To achieve this, we made extensive use of the superconformal tensor calculus from which we deduced several useful auxiliary formulae such as multiplication rules of multiplets and maps between different multiplets.

To avoid ghosts at the linearized level, we avoided terms of the form $R \square R$ and $R_{\mu \nu} \square R^{\mu \nu}$. We can easily extend our analysis and construct supersymmetric invariants that contain such terms. Models with such terms generically contain two massive gravitons one of which is a ghost. However, there are critical values of the parameters in which one or two of the massive gravitons become massless thereby avoiding the massive ghost. In particular, the tri-critical gravity model, in which both massive gravitons become massless, was studied in [19]. The tri-critical model of [19] was limited in the sense that it did not include terms cubic in the curvatures. We find that in the same way that the absence of a $S \square S$ kinetic term fixes the NMG combination of $R^{2}$ and $R_{\mu \nu} R^{\mu \nu}$ terms, the absence of a higher-derivative $S \square^{2} S$ term fixes the combination of $R \square R$ and $R_{\mu \nu} \square R^{\mu \nu}$ terms to the one corresponding to tri-critical gravity. However, we also find that it is not possible to cancel all off-diagonal terms in the supersymmetric extension of tri-critical gravity while keeping supersymmetry. ${ }^{7}$

This leads us to consider the most general model containing all possible curvature terms, with and without explicit derivatives. A particularly interesting combination of terms of mass dimension 6 is given by

$$
e^{-1} \mathcal{L}_{\text {Tri }}=R_{\mu \nu} \square R^{\mu \nu}-\frac{3}{8} R \square R-3 R_{\mu \nu} R^{\nu \rho} R_{\rho}{ }^{\mu}+\frac{5}{2} R R_{\mu \nu} R^{\mu \nu}-\frac{1}{2} R^{3} .
$$

Recently, it has been shown [21] that this combination is free from the non-linear BoulwareDeser ghosts [22]. We find that there is no supersymmetric extension of this invariant where all off-diagonal terms have been canceled. This is not surprising given the fact that this model, due to the $R \square R$ and $R_{\mu \nu} \square R^{\mu \nu}$ terms, contains a ghostly massive graviton.

It is intriguing that there are now several criteria that all hint to the same higherderivative cubic extended NMG gravity model if we restrict to terms with at most 6 derivatives and require the absence of ghosts. We are aware of the following ones:

(1) Supersymmetry In this work we have shown that supersymmetry (and requiring the absence of ghosts when linearized around an $\mathrm{AdS}_{3}$ background) leads to a unique answer for the purely gravitational terms, see eq. (5.3), of mass dimension 6 corresponding to the cubic extended NMG model.

(2) Holographic c-theorem The same combination of cubic curvature terms (5.3), also follows from considerations on the holographic c-theorem [8].

(3) Born-Infeld Gravity The cubic extended NMG model follows from a truncation of Born-Infeld gravity [6]. Unlike the previous two cases, the truncation of Born-Infeld

\footnotetext{
${ }^{7}$ This is presumably related to the fact that most likely tri-critical gravity at the nonlinear level contains ghosts [20].
} 
gravity fixes the relative coefficients between the Einstein-Hilbert, quadratic, and cubic invariants. Therefore, the holographic c-theorem and supersymmetry leads to a wider class of ghost-free invariants then Born-Infeld gravity. In other words, Born-Infeld gravity corresponds to a specific choice of relative coefficients.

(4) Absence of Boulware-Deser Ghosts There is one more criterion. Recently, it has been shown in [21] that precisely the cubic extended NMG model follows from a Chern-Simons like formulation in which it is relatively easy to show the absence of non-linear ghosts using the Hamiltonian formalism [23, 24].

It is a challenge to see whether the Born-Infeld gravity model mentioned in criterion (3) passes the tests (1), (2) and (4). As far as criterion (1) is concerned, one could repeat the analysis of this paper to one level higher. However, for an all order result presumably a better formulation of Born-Infeld supergravity using superspace techniques is needed [25]. Concerning criterion (4) it would be interesting to see whether the full Born-Infeld gravity model allows for a Chern-Simons like formulation, see also [21].

\section{Acknowledgments}

We wish to thank H. Afshar, G.Alkac, L. Basanisi and W. Merbis for useful discussions. One of us (M.O.) is funded by a grant from Groningen University and the KNAW.

Open Access. This article is distributed under the terms of the Creative Commons Attribution License (CC-BY 4.0), which permits any use, distribution and reproduction in any medium, provided the original author(s) and source are credited.

\section{References}

[1] G. Lopes Cardoso, B. de Wit and T. Mohaupt, Corrections to macroscopic supersymmetric black hole entropy, Phys. Lett. B 451 (1999) 309 [hep-th/9812082] [INSPIRE].

[2] J.M. Maldacena, The Large- $N$ limit of superconformal field theories and supergravity, Int. J. Theor. Phys. 38 (1999) 1113 [hep-th/9711200] [INSPIRE].

[3] R.C. Myers and A. Sinha, Holographic c-theorems in arbitrary dimensions, JHEP 01 (2011) 125 [arXiv: 1011.5819] [INSPIRE].

[4] E.A. Bergshoeff, O. Hohm and P.K. Townsend, Massive Gravity in Three Dimensions, Phys. Rev. Lett. 102 (2009) 201301 [arXiv:0901.1766] [INSPIRE].

[5] S. Deser, R. Jackiw and S. Templeton, Topologically Massive Gauge Theories, Annals Phys. 140 (1982) 372 [Erratum ibid. 185 (1988) 406] [INSPIRE].

[6] I. Gullu, T.C. Sisman and B. Tekin, Born-Infeld extension of new massive gravity, Class. Quant. Grav. 27 (2010) 162001 [arXiv: 1003.3935] [INSPIRE].

[7] S. Deser and G.W. Gibbons, Born-Infeld-Einstein actions?, Class. Quant. Grav. 15 (1998) L35 [hep-th/9803049] [INSPIRE].

[8] A. Sinha, On the new massive gravity and AdS/CFT, JHEP 06 (2010) 061 [arXiv: 1003.0683] [INSPIRE]. 
[9] M.F. Paulos, New massive gravity extended with an arbitrary number of curvature corrections, Phys. Rev. D 82 (2010) 084042 [arXiv: 1005.1646] [inSPIRE].

[10] R. Andringa, E.A. Bergshoeff, M. de Roo, O. Hohm, E. Sezgin et al., Massive 3D Supergravity, Class. Quant. Grav. 27 (2010) 025010 [arXiv:0907.4658] [INSPIRE].

[11] E.A. Bergshoeff, O. Hohm, J. Rosseel, E. Sezgin and P.K. Townsend, More on Massive 3D Supergravity, Class. Quant. Grav. 28 (2011) 015002 [arXiv:1005.3952] [INSPIRE].

[12] T. Nutma, Polycritical Gravities, Phys. Rev. D 85 (2012) 124040 [arXiv:1203.5338] [INSPIRE].

[13] S.J. Gates, M.T. Grisaru, M. Roček and W. Siegel, Superspace Or One Thousand and One Lessons in Supersymmetry, hep-th/0108200 [INSPIRE].

[14] P. van Nieuwenhuizen, D=3 Conformal Supergravity and Chern-Simons Terms, Phys. Rev. D 32 (1985) 872 [INSPIRE].

[15] T. Uematsu, Structure of $N=1$ Conformal and Poincaré Supergravity in (1+1)-dimensions and (2+1)-dimensions, Z. Phys. C 29 (1985) 143 [InSPIRE].

[16] T. Uematsu, Constraints and Actions in Two-dimensional and Three-dimensional $N=1$ Conformal Supergravity, Z. Phys. C 32 (1986) 33 [INSPIRE].

[17] P.S. Howe and R.W. Tucker, Local Supersymmetry in (2+1)-Dimensions. 1. Supergravity and Differential Forms, J. Math. Phys. 19 (1978) 869 [INSPIRE].

[18] I. Gullu, T.C. Sisman and B. Tekin, c-functions in the Born-Infeld extended New Massive Gravity, Phys. Rev. D 82 (2010) 024032 [arXiv: 1005.3214] [INSPIRE].

[19] E.A. Bergshoeff, S. de Haan, W. Merbis, J. Rosseel and T. Zojer, On Three-Dimensional Tricritical Gravity, Phys. Rev. D 86 (2012) 064037 [arXiv: 1206.3089] [InSPIRE].

[20] L. Apolo and M. Porrati, Nonlinear Dynamics of Parity-Even Tricritical Gravity in Three and Four Dimensions, JHEP 08 (2012) 051 [arXiv:1206.5231] [INSPIRE].

[21] H.R. Afshar, E.A. Bergshoeff and W. Merbis, Extended massive gravity in three dimensions, arXiv: 1405.6213 [INSPIRE].

[22] D.G. Boulware and S. Deser, Can gravitation have a finite range?, Phys. Rev. D 6 (1972) 3368 [INSPIRE].

[23] O. Hohm, A. Routh, P.K. Townsend and B. Zhang, On the Hamiltonian form of $3 D$ massive gravity, Phys. Rev. D 86 (2012) 084035 [arXiv:1208.0038] [INSPIRE].

[24] E.A. Bergshoeff, O. Hohm, W. Merbis, A.J. Routh and P.K. Townsend, The Hamiltonian Form of Three-Dimensional Chern-Simons-like Gravity Models, arXiv:1402.1688 [INSPIRE].

[25] S.J. Gates Jr. and S.V. Ketov, 4-D, $N=1$ Born-Infeld supergravity, Class. Quant. Grav. 18 (2001) 3561 [hep-th/0104223] [INSPIRE]. 